\title{
Role of Oxidant Stress in the Permeability Transition Induced in Rat Hepatic Mitochondria by Hydrophobic Bile Acids
}

\author{
RONALD J. SOKOL, MICHAEL S. STRAKA, ROLF DAHL, MICHAEL W. DEVEREAUX, \\ BARUCH YERUSHALMI, ERIC GUMPRICHT, NANCY ELKINS, AND GREGORY EVERSON \\ Pediatric Liver Center, Section of Pediatric Gastroenterology, Hepatology and Nutrition, Department of \\ Pediatrics [R.J.S., M.S.S., R.D., M.W.D., B.Y., E.G.], Section of Hepatology, Department of Medicine, and \\ the Hepatobiliary Research Center [G.E.], and the Pediatric General Clinical Research Center [R.J.S.], \\ University of Colorado School of Medicine, Denver, Colorado 80262, U.S.A., and The Children's Hospital, \\ Denver, Colorado 80218, U.S.A., and Webb-Waring Institute, Denver, Colorado 80262, U.S.A. [N.E.]
}

\begin{abstract}
Hydrophobic bile acids may cause hepatocellular necrosis and apoptosis during cholestatic liver diseases. The mechanism for this injury may involve mitochondrial dysfunction and the generation of oxidant stress. The purpose of this study was to determine the relationship of oxidant stress and the mitochondrial membrane permeability transition (MMPT) in hepatocyte necrosis induced by bile acids. The MMPT was measured spectrophotometrically and morphologically in rat liver mitochondria exposed to glycochenodeoxycholic acid (GCDC). Freshly isolated rat hepatocytes were exposed to GCDC and hepatocellular necrosis was assessed by lactate dehydrogenase release, hydroperoxide generation by dichlorofluorescein fluorescence, and the MMPT in cells by JC1 and tetramethylrhodamine methylester fluorescence on flow cytometry. GCDC induced the MMPT in a dose- and $\mathrm{Ca}^{2+}$-dependent manner. Antioxidants significantly inhibited the GCDC-induced MMPT and the generation of hydroperoxides in isolated mitochondria. Other detergents failed to induce the MMPT and a calpain-like protease inhibitor had no effect on the GCDC-induced MMPT. In isolated rat hepatocytes, GCDC induced the MMPT, which was inhibited by antioxidants. Blocking the MMPT in hepatocytes reduced hepatocyte necrosis and oxidant stress caused by GCDC. Oxidant stress, and not detergent effects or the stimulation of calpain-like proteases,
\end{abstract}

\section{ABSTRACT}

mediates the GCDC-induced MMPT in hepatocytes. We propose that reducing mitochondrial generation of reactive oxygen species or preventing increases in mitochondrial $\mathrm{Ca}^{2+}$ may protect the hepatocyte against bile acid-induced necrosis. (Pediatr Res 49: 519-531, 2001)

Abbreviations
Cbz-Leu-Leu-Tyr, $N$-carbonylbenzyloxy-L-leucyl-L-leucyl-L-
tyrosine, diazomethyl ketone
CHAPS, 3-[(3-cholamidopropyl)dimethyl, ammonio]-1-
propanesulfonate
CMC, critical micellar concentration
GCDC, glycochenodeoxycholic acid
FACS, fluorescence-activated cell sorter
FCCP, carbonyl cyanide p-trifluoromethoxyphenylhydrazone
MMPT, mitochondrial membrane permeability transition
ROS, reactive oxygen species
TBARS, thiobarbituric acid-reactive substances
TFP, trifluoperazine
TMRM, tetramethylrhodamine methylester
Triton X-100, $t$-octylphenoxypolyethoxyethanol
$\Delta \psi$, mitochondrial electrochemical gradient

A factor implicated in the pathogenesis of cholestatic liver injury is the hepatic retention of hydrophobic bile acids, such

Received August 29, 2000; accepted November 24, 2000.

Correspondence and reprint requests: Ronald J. Sokol, M.D., Professor of Pediatrics, Pediatric Liver Center and Liver Transplantation Program, Box 290, The Children's Hospital, 1056 East 19th Avenue, Denver, CO 80218-1088, U.S.A.; e-mail: Sokol.Ronald@tchden.org

Supported in part by grants from the National Institutes of Health (RO1DK38446 and IP30 DK34914) and the Abbey Bennett Liver Research Fund.

Presented in part at the American Association for the Study of Liver Diseases Annual Meeting, Chicago, IL, U.S.A., November 1996, and published in abstract form (Hepatology 1996: 24:237A) as conjugates of chenodeoxycholic acid (CDC) (1-3). Although hydrophobic bile acids cause injury to isolated hepatocytes (4), cultured hepatocytes (5), and the intact liver (6), the mechanisms of this toxicity are not fully understood. Both hepatocellular necrosis at higher bile acid concentrations (4) and apoptosis at lower concentrations (7) have been demonstrated and are proposed as playing a role in cholestatic liver injury. Hepatocyte necrosis is characterized by cellular swelling, loss of mitochondrial respiratory function, depleted cellular ATP levels, and formation of plasma membrane blebs that rupture and release cellular contents $(8,9)$. In cholestatic liver 
disorders of infancy, massive swelling of hepatocytes that contain accumulated bile and elevated serum hepatocellular aminotransferase enzymes are characteristic findings (10). Similar histologic changes in hepatocytes have been described in liver from adults with cholestasis, so-called "feathery degeneration" (11). Thus, histologic features of hepatocyte necrosis seem to be common in the liver of humans with cholestatic disorders.

Recent studies have suggested that oxidant stress may play an important role in the pathogenesis of hepatic injury during cholestasis $(6,12-16)$. Supporting this proposed mechanism is the observation that $\alpha$-tocopherol, the major membraneassociated, lipid-soluble antioxidant, reduces both the generation of ROS and injury to hepatocytes exposed to hydrophobic bile acids $(17,18)$ and in the intact rat infused with bile acids (6). Several lines of evidence also support hepatic mitochondria as a major source of the oxidant stress imposed by hydrophobic bile acids, including observations that hepatic mitochondria undergo lipid peroxidation during experimental cholestasis and bile acid toxicity $(6,16)$, that hydrophobic bile acids impair respiration and electron transport in hepatic mitochondria (19), and that hydrophobic bile acids stimulate the generation of ROS by isolated hepatic mitochondria (18).

More recently, hydrophobic bile acids have been shown to induce the MMPT in hepatic mitochondria $(20,21)$. The MMPT is a rapid increase in the permeability of the inner mitochondrial membrane to solutes of molecular mass $<1500$ $\mathrm{D}$ that results in collapse of the electrochemical gradient $\left(\Delta \psi_{\mathrm{i}}\right)$ across the inner membrane, uncoupling of oxidative phosphorylation, and colloid-osmotic swelling of mitochondria (22-24). Induction of the MMPT precedes the onset of cell necrosis (22, $23,25,26)$ and may also be central to the process of apoptosis $(27,28)$. The MMPT is mediated by the opening of a transmembrane proteinaceous megachannel, the mitochondrial permeability pore, which shares electrophysiological properties with the voltage-dependent anion channel $(29,30)$ and includes the mitochondrial adenine nucleotide translocator as a key component $(31,32)$. The peptide, cyclosporin A, has been shown to bind to mitochondrial cyclophilin (28), which specifically inhibits the opening of the permeability pore and prevents the MMPT $(25,32-34)$. The role of the MMPT in the mechanistic pathway that leads to cellular necrosis may involve $\operatorname{NADP}(\mathrm{H})$ oxidation, a reduced capacity for oxidative phosphorylation and depletion of cellular ATP, alterations in cellular calcium homeostasis, and plasma membrane structural changes $(4,26,27)$. Several of these events have been observed in bile acid-induced hepatocyte necrosis $(4,8)$, however, the precise mechanisms by which bile acids induce the MMPT and subsequent hepatocyte necrosis are not well characterized. The determinant of whether necrosis or apoptosis will occur after induction of the MMPT may be the residual cellular ATP levels. If a large number of mitochondria in a given cell undergo the permeability transition and cellular ATP levels become depleted, necrosis is favored, whereas maintenance of ATP levels when a fewer number of mitochondria undergo the MMPT will favor apoptosis (27). Recent studies suggest that bile acid-induced apoptosis may also involve mitochondrial proteases (20), protein kinase C (35), the Fas signaling path- way (36), translocation of Bax to mitochondria (37), or downstream caspases (36). Several of these pathways may induce or regulate apoptosis by activation or inhibition of the MMPT.

In this study, we further explored the mechanistic role of oxidant stress in hepatocyte necrosis caused by bile acids. We postulated that mitochondrial respiratory dysfunction caused by accumulated hydrophobic bile acids generated increased ROS that induced the MMPT, triggering irreversible events that led to cellular necrosis. Therefore, the major objective of this study was to provide insight into the interrelationship of ROS generation and induction of the MMPT in mitochondria and hepatocytes exposed to concentrations of hydrophobic bile acids that caused cellular necrosis. The specific aims of this study were (1) to understand factors that regulate the MMPT induced by GCDC, the hydrophobic bile acid implicated in the pathogenesis of cholestatic liver disease; (2) to determine whether detergent properties of bile acids play a role in MMPT induction; (3) to determine the role of oxidative stress in the bile acid-induced MMPT; and (4) to determine whether the MMPT occurred in hepatocytes undergoing bile acid-induced necrosis and if blocking the MMPT protected isolated hepatocytes from bile acid-induced necrosis. The results of this study strongly support an important role for ROS generation in the induction of the MMPT and cellular necrosis caused by hydrophobic bile acids.

\section{MATERIALS AND METHODS}

\section{Materials}

All chemicals were obtained from Sigma Chemical Co. (St. Louis, MO, U.S.A.) and were of analytical grade, except where otherwise noted.

\section{Isolation of Rat Liver Mitochondria}

Rat liver mitochondria were isolated from adult male Sprague Dawley rats (150-200 g weight), which were maintained on a 12-h light-dark cycle and fed standard laboratory rat chow. Humane care was given to all experimental animals and this study was approved by the Institutional Animal Care and Use Committee of the University of Colorado Health Sciences Center. Mitochondria were isolated by differential centrifugation as previously described (16), with the following modifications: freshly isolated livers were rinsed in $250 \mathrm{mM}$ sucrose, $1 \mathrm{mM}$ EGTA, pH 7.4. Livers were homogenized in buffer containing $220 \mathrm{mM}$ mannitol, $70 \mathrm{mM}$ sucrose, $10 \mathrm{mM}$ HEPES, 1 mM EGTA, pH 7.4. Homogenates were centrifuged for $10 \mathrm{~min}$ at $400 \times g$; the supernatant was then centrifuged at $7000 \times g$ for $10 \mathrm{~min}$. The resulting pellet was resuspended in $1 \mathrm{~mL}$ of wash buffer and layered onto a preformed gradient of $75 \%$ sucrose $/ 25 \%$ Percoll and centrifuged at $36,000 \times g$ for 26 min and then washed two times in wash buffer. Wash buffer consisted of $100 \mathrm{mM} \mathrm{KCl}, 5 \mathrm{mM} 3$-[N-morpholino] propanesulfonic acid (MOPS), 1 mM EGTA, pH 7.4, treated with 1\% (wt:vol) iminodiacetic acid immobilized on crosslinked polystyrene (Chelex 100). The resultant purified mitochondria were then resuspended in final buffer containing $125 \mathrm{mM}$ sucrose, 50 
$\mathrm{mM} \mathrm{KCl}, 5 \mathrm{mM}$ HEPES, $2 \mathrm{mM} \mathrm{KH}{ }_{2} \mathrm{PO}_{4}$, $\mathrm{pH}$ 7.4, treated with 1\% Chelex 100.

\section{Measurement of the Mitochondrial Membrane Permeability Transition}

The MMPT was measured by the spectrophotometric method described by Pastorino et al. (25) as modified by Botla et al. (21) and by transmission electron microscopy. In the spectrophotometric assay, the MMPT was equated with rapid, high-amplitude swelling of mitochondria, taking advantage of the linear relationship between average mitochondrial volume and the reciprocal of absorbance (38). Thus, mitochondrial swelling was monitored as a decrease in OD. Mitochondria were diluted to $1 \mathrm{mg}$ protein $/ \mathrm{mL}$ in respiration buffer containing $125 \mathrm{mM}$ sucrose, $100 \mathrm{mM} \mathrm{NaCl}$, and $10 \mathrm{mM}$ MOPS, $\mathrm{pH}$ 7.4 , treated with $1 \%$ Chelex 100 . The OD at $540 \mathrm{~nm}$ was monitored at $25^{\circ} \mathrm{C}$ for a total of $10 \mathrm{~min}$ in a Perkin-Elmer (Norwalk, CT, U.S.A.) model Lambda-2 spectrophotometer. Experiments were conducted using $1.5 \mathrm{~mL}$ mitochondrial suspension that were preincubated from $t=-10 \min$ to $t=-5$ min with either $5 \mu \mathrm{M}$ cyclosporin A (Sandimmune, a gift of the Sandoz Research Institute, East Hanover, NJ, U.S.A.), the calpain protease inhibitor Cbz-Leu-Leu-Tyr (Molecular Probes, Eugene, OR, U.S.A.), or the indicated concentrations of the antioxidants R,R,R- $\alpha$-tocopherol (Fisher Scientific, Pittsburgh, PA, U.S.A.), sodium ascorbate, the coenzyme Q analogue, idebenone (Takeda Laboratories, Tokyo, Japan), the peroxidase ebselen (Sigma Chemical Co. St. Louis, MO, U.S.A.), or corresponding volumes of their respective solvents. At $\mathrm{t}=-5 \mathrm{~min}, \mathrm{CaCl}_{2}$ was added and respiration via complex I was initiated by the addition of glutamate and malate (final concentration $=1 \mathrm{mM}$ ) or via complex II by the addition of sodium succinate $(5 \mathrm{mM})$. In the $\mathrm{Ca}^{2+}$-dependence experiments, the final concentration of $\mathrm{Ca}^{2+}$ varied from 0 to 150 $\mu \mathrm{M}$, whereas in all other experiments the final concentration of $\mathrm{Ca}^{2+}$ was $100 \mu \mathrm{M}$. At $\mathrm{t}=-2 \mathrm{~min}$ in all experiments, rotenone ( $5 \mu \mathrm{M}$ in dimethylformamide), an inhibitor of complex I, was added to the suspension, as described by others $(7,25)$. At $t=$ $0 \mathrm{~min}$, the sodium salt of GCDC dissolved in the respiration buffer was added; in dose-response experiments, the final concentration of GCDC ranged from 25 to $400 \mu \mathrm{M}$, and for all other experiments, 50-200 $\mu \mathrm{M}$ GCDC was used. Control experiments demonstrated that corresponding volumes of solvent vehicles alone had no effect on the MMPT. In a separate series of experiments, antioxidants or cyclosporin A were added to the mitochondrial suspension $1 \mathrm{~min}$ after the addition of GCDC rather than during the preincubation. In other experiments, the detergents CHAPS and Triton X-100 were used in place of GCDC as inducers of the MMPT. Final concentrations of CHAPS ranged from 0 to $800 \mu \mathrm{M}$, whereas those of Triton $\mathrm{X}-100$ were 0 to $200 \mu \mathrm{M}$. In an additional set of experiments, the effect of oxygen-free buffer on the GCDC-induced MMPT was determined. Hepatic mitochondria were isolated as above, however, they were resuspended in buffer treated with $100 \%$ nitrogen gas and maintained under an atmosphere of $100 \%$ nitrogen during induction of the MMPT, which was conducted as described above.
In three experiments, the MMPT was analyzed morphologically by transmission electron microscopy. Aliquots of mitochondria were removed at various time points throughout the MMPT experiment, fixed immediately in $2.5 \%$ glutaraldehyde, centrifuged into a pellet, and then postfixed in $2 \% \mathrm{OsO}_{4}$. After dehydration, the mitochondria were embedded in epoxy resin. Ultrathin sections were stained with uranyl acetate and lead citrate and examined by transmission electron microscopy with a Philips (Eindhoven, Netherlands) CM10 electron microscope. The pellet was sampled by systematically taking four electron micrographs, each of different regions of the pellet, starting with the bottom and sampling to the top. At least 100 mitochondria per condition were measured. After electronically scanning the micrographs, the cross-sectional area per mitochondrion was calculated using the National Institutes of Health Image 1.60 software.

\section{Measurement of Hydroperoxide Generation in Isolated Mitochondria}

To determine whether GCDC induced significant generation of ROS during the time course of MMPT induction, hydroperoxide generation was measured in isolated mitochondria by the fluorescent probe, dichlorofluorescein (DCFein), as previously described (20). Dichlorofluorescin-diacetate (DCF-DA) is taken up by mitochondria (or hepatocytes) and intramitochondrial (or intracellular) esterases hydrolyze the acetate esters, trapping free dichlorofluorescin (DCF) inside mitochondria (or cells). The nonfluorescent DCF is converted to the fluorescent DCFein by intramitochondrial or intracellular hydroperoxides (hydrogen peroxide and lipid hydroperoxides). Because DCF-DA does not itself react with hydroperoxides, only intramitochondrial (or intracellular) hydroperoxides are detected by this method. A solution of DCF-DA $(27 \mathrm{mM})$ in dimethylformamide (DMF) was made up fresh for each experiment. The final mitochondrial pellet was resuspended in wash buffer, loaded with DCF-DA $(8 \mu \mathrm{M})$ at $28^{\circ} \mathrm{C}$ for $30 \mathrm{~min}$, washed twice with wash buffer and centrifuged at $10,000 \times g$ for 10 min, and then resuspended in $20 \mathrm{~mL}$ of final buffer. Aliquots of mitochondria were removed, centrifuged, and resuspended in $30 \mathrm{~mL}$ of respiration buffer. Mitochondria were then preincubated with the antioxidants $\alpha$-tocopherol $(100 \mu \mathrm{M})$, idebenone $(10 \mu \mathrm{M})$, or sodium ascorbate $(1 \mathrm{mM})$, or the MMPT blockers cyclosporin $\mathrm{A}(1 \mu \mathrm{M})$ and bongkrekic acid $(5 \mu \mathrm{M})$, or their respective vehicles for $10 \mathrm{~min}$. Mitochondria were then incubated with $\mathrm{CaCl}_{2}(100 \mu \mathrm{M})$, succinate $(5 \mathrm{mM})$, and rotenone (5 $\mu \mathrm{M})$ in the same manner as for the MMPT assay, followed by the addition of GCDC to a final concentration of $0-100 \mu \mathrm{M}$. Aliquots of mitochondria ( $3 \mathrm{~m}$.) were removed at $0,1,3,5$, and $10 \mathrm{~min}$, and DCFein fluorescence $(490 \mathrm{~nm}$ excitation and 520 $\mathrm{nm}$ emission wavelengths) was recorded on a Perkin-Elmer MPF-66 fluorimeter as a measure of hydroperoxide generation (18). Results were compared with a standard curve using $2^{\prime} 7$ '-dichlorofluorescein as the standard and were expressed as pmol of DCFein per milligram mitochondrial protein present at each time point. The average protein content of the final mitochondrial suspension, determined by the bicinchoninic acid protein assay (Sigma Chemical Co.), was $\cong 1.0 \mathrm{mg} / \mathrm{mL}$. 
To determine the effect of varying concentrations of cyclosporin A on the MMPT and ROS generation, isolated mitochondria were preincubated with cyclosporin A $(0,0.0025$, $0.005,0.01,0.05,0.125,0.250,0.50,1.0$, and $5.0 \mu \mathrm{M})$ and the MMPT initiated by $100 \mu \mathrm{M}$ GCDC during succinatestimulated respiration. MMPT was measured by the absorbance method and ROS generation by the DCFein assay at 0 , $1,3,5$, and 10 min after the addition of the GCDC.

\section{Effect of MMPT Blockers on Hepatocyte Toxicity and ROS Generation}

Isolated hepatocyte studies. To determine whether blocking the MMPT reduced cell necrosis and oxidant stress of hepatocytes exposed to hydrophobic bile acids, experiments were conducted in fresh hepatocytes isolated from adult Sprague Dawley rats (weight $150-200$ g) by a recirculating collagenase perfusion technique previously described $(17,18)$, and maintained in suspension in incubation buffer of Kreb-Ringers HEPES (KRH) buffer $(115 \mathrm{mM} \mathrm{NaCl}, 5 \mathrm{mM} \mathrm{KCl}, 1 \mathrm{mM}$ $\mathrm{KH}_{2} \mathrm{PO}_{4}, 1.2 \mathrm{mM} \mathrm{MgSO}_{4}$, and $25 \mathrm{mM} \mathrm{Na}{ }^{+}$HEPES, $\mathrm{pH}$ 7.4) containing $0.2 \%$ BSA. Freshly isolated rat hepatocytes, rather than primary cultured hepatocytes or hepatoma cell lines, were used in this study so that bile acid uptake (39) and antioxidant defenses of the cells would be preserved (40), thus representing hepatocytes in the intact liver. Following isolation, hepatocyte viability was greater than $95 \%$ by trypan blue exclusion (17). Cells were then preincubated with the MMPT blockers, cyclosporin A $(5 \mu \mathrm{M})$, and trifluoperazine (TFP; $10 \mu \mathrm{M})$, or the appropriate vehicle for $10 \mathrm{~min}$ and then exposed to 0 or 500 $\mu \mathrm{M}$ GCDC for $4 \mathrm{~h}$. Aliquots of cells were removed hourly for $4 \mathrm{~h}$ and then analyzed for cellular necrosis by release of lactate dehydrogenase (LDH) (18), lipid peroxidation by the thiobarbituric acid reacting substances (TBARS) assay $(17,18)$, and ROS (hydroperoxides) generation by the DCFein assay (18). For the TBARS assay, $0.2 \mathrm{~mL}$ of hepatocyte suspension was added to $0.5 \mathrm{~mL}$ of trichloroacetic acid $(10 \% \mathrm{wt} / \mathrm{vol})$ and 50 $\mu \mathrm{L}$ of butylated hydroxytoluene $(2 \% \mathrm{wt} / \mathrm{vol})$, vortexed, and centrifuged at $1295 \times g$ for $10 \mathrm{~min}$. The supernatant was added to $1 \mathrm{~mL}$ of TBA $(0.67 \% \mathrm{wt} / \mathrm{vol})$ and heated in a water bath to $100^{\circ} \mathrm{C}$ for $15 \mathrm{~min}$. After cooling to room temperature and centrifugation $(1000 \times g$ for $10 \mathrm{~min})$, absorbance at $532 \mathrm{~nm}$ was determined on the supernatant and compared with a standard curve using 1,1,3,3-tetraethoxypropane as the standard. TBARS were expressed as nmol $/ 10^{6}$ cells.

For the hydroperoxide assay (18), hepatocytes were preloaded with $8 \mu \mathrm{M}$ DCF-DA at $37^{\circ} \mathrm{C}$ for $30 \mathrm{~min}$, washed twice by centrifugation at $50 \times g$ for $1 \mathrm{~min}$, and resuspended in incubation buffer (KRH $+0.2 \% \mathrm{BSA})$. The cells were then preincubated with and without the MMPT blockers for $10 \mathrm{~min}$, and then the indicated concentrations of GCDC were added. Aliquots of cells $(0.8 \mathrm{~mL})$ were removed each hour, added to $2.2 \mathrm{~mL}$ of incubation buffer and analyzed for DCF fluorescence as described for mitochondria, and expressed as pmol $/ 10^{6}$ hepatocytes compared with a standard curve generated by 2'7'-dichlorofluorescein.

Bile acid analysis. To determine whether cyclosporin A had any significant effect on the uptake or retention of GCDC by the isolated hepatocytes, concentrations of conjugated and unconjugated species of chenodeoxycholic acid (CDC) were measured in aliquots of hepatocytes after $0,1,2$, and $4 \mathrm{~h}$ of incubation with 0 or $500 \mu \mathrm{M}$ GCDC with and without cyclosporin A and TFP. Briefly, $20 \times 10^{6}$ hepatocytes were removed at the indicated time points, washed rapidly with icecold buffer and centrifuged at $40 \times g$ for 1 min three times (only initial wash contained 2\% BSA to remove adherent bile acids) and then stored at $-70^{\circ} \mathrm{C}$. Free and conjugated bile acids were then measured by gas chromatography-mass spectrometry by modification of previously described techniques (41). To each sample of hepatocytes was added an internal standard (7-alpha, 12 alpha, dihydroxy-5 beta cholanic acid) in butanol. The samples and appropriate standards were incubated with $2 \mathrm{~N}$ sodium hydroxide at $80^{\circ} \mathrm{C}$ for $1.5 \mathrm{~h}$, cooled to room temperature, $\mathrm{pH}$ adjusted to 8.0 , and trypsinized at $37^{\circ} \mathrm{C}$ for $2 \mathrm{~h}$. Cooled samples were then eluted through C18 Sep-paks (Waters Corp., Milford, MA, U.S.A.) using $85 \%$ methanol in water, the eluent was then evaporated by $\mathrm{N}_{2}$ gas in a $60^{\circ} \mathrm{C}$ water bath. Conjugates from the residue were then hydrolyzed by fresh cholylglycine hydrolase at $37^{\circ} \mathrm{C}$ overnight. Free bile acids were then extracted in diethyl ether after acidification, followed by methylation and the formation of trimethylsilyl ethers. The residue was extracted in hexane and injected into a Hewlett-Packard 5790 gas chromatograph (Hewlett-Packard Co., Wilmington, DE, U.S.A.) with a flame ionization detector equipped with a $30 \mathrm{~m}$ DB-1 capillary column (J\&W Scientific, Folsom, CA, U.S.A.) with internal diameter of $0.25 \mathrm{~mm}$ and a film thickness of $0.25 \mu \mathrm{m}$ at $215-290^{\circ} \mathrm{C}$. Selected ion monitoring was performed on a Hewlett-Packard 5970-A mass selective detector. Results were expressed as nanmole of CDC per milligram cellular protein.

Flow cytometry studies. Finally, FACS analysis, using the fluorescent mitochondrial probes, TMRM, and JC-1, were used to verify that GCDC dissipated the mitochondrial membrane $\Delta \psi$ in isolated hepatocytes, indicating the opening of the permeability pore, and the effect of antioxidants on the $\Delta \psi$. TMRM accumulates in mitochondria in proportion to the mitochondrial membrane $\Delta \psi$. JC-1, at relatively low concentrations, exists in a monomeric form that fluoresces at $527 \mathrm{~nm}$; when concentrated by actively respiring mitochondria, JC-1 aggregates form that fluoresce at $590 \mathrm{~nm}$ (42). The intensity of fluorescence at $590 \mathrm{~nm}$ is proportional to the $\Delta \psi$, which indicates a closed permeability transition pore. Upon induction of the MMPT, dissipation of the $\Delta \psi$ prevents the formation of JC-1 aggregates and diminishes the fluorescence at $590 \mathrm{~nm}$.

Hepatocytes were exposed to $500 \mu \mathrm{M}$ GCDC in KRH + $0.2 \% \mathrm{BSA}$, aliquots were removed at $0,1,2,3$, and $4 \mathrm{~h}$ and then loaded with JC-1 $(7.6 \mu \mathrm{M})$ or TMRM $(1 \mu \mathrm{M})$ in the same buffer for $15 \mathrm{~min}$ at $22^{\circ} \mathrm{C}$ in the dark. After washing the cells with buffer at $4^{\circ} \mathrm{C}$, the hepatocytes were analyzed by flow cytometry and fluorescence of the probes was measured at the appropriate wavelengths with a Bectin Dickinson FACS Calibur (Bectin Dickinson Immunocytometry Systems, San Jose, CA, U.S.A.) using CELLQuest software. Ten thousand cells were analyzed at each time point. The JC-1 monomers were detected at a peak fluorescence of $530 \mathrm{~nm}$, and JC-1 aggregates and TMRM at $575 \mathrm{~nm}$. All were excited with the $488 \mathrm{~nm}$ line 
of an argon ion laser at $15 \mathrm{~mW}$. In some experiments, cells were preincubated for $15 \mathrm{~min}$ with $\alpha$-tocopherol $(250 \mu \mathrm{M})$ or idebenone $(100 \mu \mathrm{M})$ before exposure to GCDC and TMRM loading. That the peak of fluorescence measured was due to mitochondrial accumulation of each fluorescent probe was confirmed by experiments that showed that $3 \mathrm{~h}$ of exposure to FCCP $(250 \mathrm{nM})$ or valinomycin $(100 \mathrm{nM})$, two compounds that dissipate mitochondrial $\Delta \psi$, resulted in the loss of fluorescence of JC-1 and TMRM.

\section{Statistical Analysis}

Statistical comparisons among experimental groups were conducted by the ANOVA with the Schefe test or the $t$ test. A $p$ value of $<0.05$ was considered statistically significant. All values are expressed as the mean $\pm \mathrm{SE}$.

\section{RESULTS}

\section{The GCDC-Induced MMPT is Concentration, Respiratory Substrate, $\mathrm{Ca}^{2+}$, and Oxygen-Dependent}

In the spectrophotometric MMPT assay, mitochondrial swelling results in a progressive decrease in absorbance (OD) at $540 \mathrm{~nm}$, which can be quantitated as the $\triangle \mathrm{OD}$ (change in absorbance units) from the time of GCDC addition at $\mathrm{t}=0 \mathrm{~min}$ to the termination of measurement at $t=+5 \mathrm{~min}$. In the first series of experiments, we examined the effect of GCDC concentration on the MMPT in the presence of $100 \mu \mathrm{M} \mathrm{CaCl}_{2}$ and during respiration stimulated by either site I (glutamate/malate) or site II (succinate) electron donors. The magnitude of the MMPT was dependent on the concentration of GCDC during glutamate/malate-stimulated respiration with relatively high concentrations $(100-400 \mu \mathrm{M})$ of GCDC necessary for MMPT induction (Fig. 1A), confirming the observations of others (21). However, during succinate-stimulated respiration, substantially lower concentrations of GCDC $(25-50 \mu \mathrm{M})$ led to significant amplitude of the MMPT (Fig. 1B). The magnitude of the MMPT was nearly linear with respect to the concentration of GCDC (Table 1). In the absence of respiratory substrates, the MMPT could not be induced by GCDC (Fig. 2). Thus, electron flow and respiration of mitochondria were essential prerequisites for the GCDC induction of the MMPT.

Experiments were then conducted in oxygen-free buffer to determine whether the GCDC-induced MMPT was dependent of the presence of oxygen, which is required for the generation of ROS. With succinate as the electron donor, the magnitude of the MMPT induced by $100 \mu \mathrm{M}$ GCDC in oxygen-free buffer was reduced by over $75 \%$ (Fig. 2). Cyclosporin A and the antioxidant $\alpha$-tocopherol continued to suppress this small residual MMPT (data not shown), confirming that this limited swelling of the mitochondria in oxygen-free buffer was caused by the permeability pore and was related to residual generation of ROS.

Because previous work by others had indicated that $\mathrm{Ca}^{2+}$ is required for induction of the MMPT by other compounds (22, 23 ), we examined the effect of varying the concentration of $\mathrm{Ca}^{2+}$ on the MMPT induced by $200 \mu \mathrm{M}$ GCDC during glutamate/malate-stimulated respiration. In these experiments, $\mathrm{Ca}^{2+}$
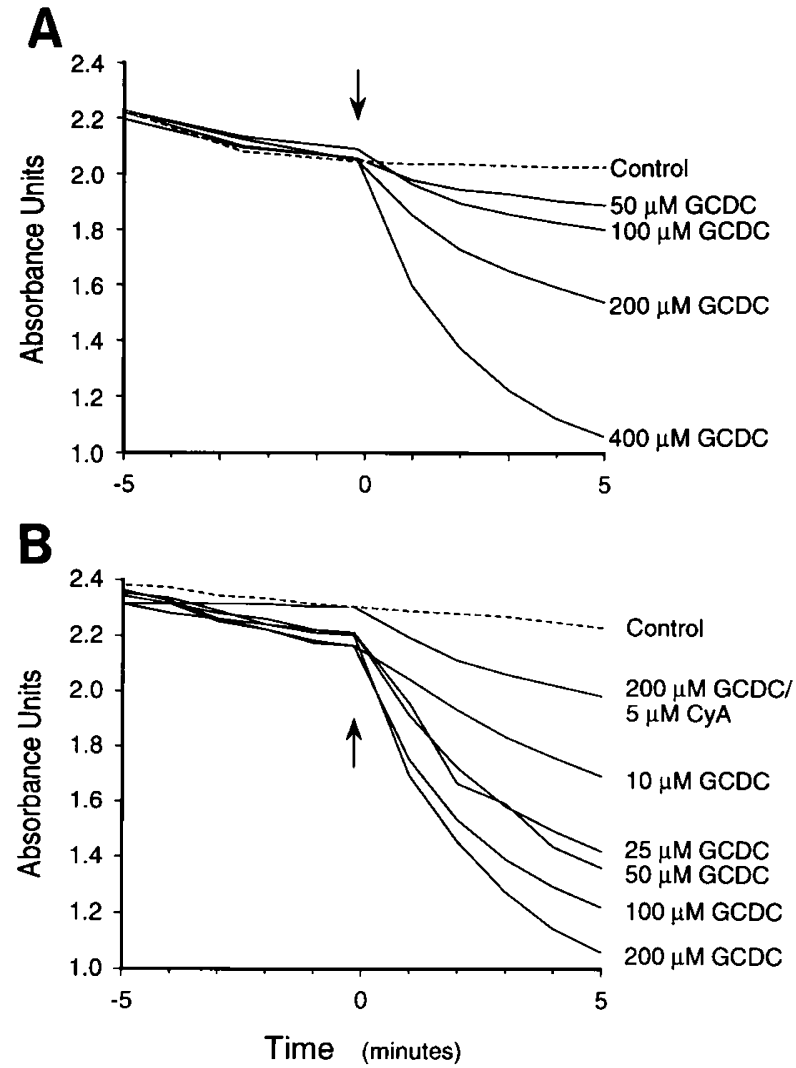

Figure 1. Relationship between concentration of GCDC and induction of MMPT in isolated rat liver mitochondria. Suspension of freshly isolated rat liver mitochondria ( $1 \mathrm{mg}$ protein $/ \mathrm{mL}$ ) in buffer containing $125 \mathrm{mM}$ sucrose, $100 \mathrm{mM} \mathrm{NaCl}, 100 \mu \mathrm{M} \mathrm{CaCl}_{2}$, and $10 \mathrm{mM} \mathrm{MOPS}, \mathrm{pH}$ 7.4, was incubated at $25^{\circ} \mathrm{C}$ for $10 \mathrm{~min}$, with the additions of $1 \mathrm{mM}$ glutamate/malate $(A)$ or $5 \mathrm{mM}$ succinate $(B)$ at $-5 \mathrm{~min}, 5 \mu \mathrm{M}$ rotenone at $-3 \mathrm{~min}$, and varying concentrations $(0-400 \mu \mathrm{M})$ of GCDC at 0 min (denoted by vertical arrow). High-amplitude swelling of mitochondria was measured by monitoring the absorbance at 540 $\mathrm{nm}$ spectrophotometrically, with a decrease in absorbance representing the MMPT. Note the higher amplitude of mitochondrial swelling at lower concentrations of GCDC when succinate was used as the respiratory substrate. Mitochondria preincubated with $5 \mu \mathrm{M}$ cyclosporin A (CyA) and exposed to $200 \mu \mathrm{M}$ GCDC showed inhibition of MMPT induction $(B)$. Values are mean of four experiments.

was required for MMPT induction (Fig. 3). Above $100 \mu \mathrm{M}$ $\mathrm{Ca}^{2+}$, the magnitude of the MMPT did not increase significantly, indicating that the $\mathrm{Ca}^{2+}$ dependence was apparently saturable. Preincubation of mitochondria with $5 \mu \mathrm{M}$ cyclosporin A completely abolished the MMPT induced by $200 \mu \mathrm{M}$ GCDC (Fig. 3), even in the presence of $150 \mu \mathrm{M} \mathrm{Ca}{ }^{2+}$, confirming that the mitochondrial swelling was in response to the opening of the mitochondrial permeability pore $(25,29,33$, $34,43)$. Similar results were obtained with succinate as the respiratory substrate (data not shown).

\section{Antioxidants Attenuate the GCDC-Induced MMPT and Hydroperoxide Generation in Isolated Mitochondria}

We next examined the effect on the MMPT of a series of antioxidants that scavenge ROS. In these experiments, mitochondrial suspensions were preincubated from $t=-10 \mathrm{~min}$ to $\mathrm{t}=0$ with the indicated concentrations of $\alpha$-tocopherol, idebenone, or ascorbate or a similar volume of the vehicle DMSO, 
Table 1. Effect of detergents on induction of MMPT

\begin{tabular}{cccc}
\hline Detergent & $\begin{array}{c}\text { Concentration } \\
(\mu \mathrm{M})\end{array}$ & Other compound & $\Delta \mathrm{OD}^{*}$ \\
\hline GCDC & 0 & & 0.018 \\
& 50 & & 0.168 \\
& 100 & & 0.287 \\
& 200 & & 0.509 \\
& 400 & & 0.997 \\
& 200 & Cyclosporin A, $5 \mu \mathrm{M}$ & 0.086 \\
CHAPS & 200 & $\alpha$-Tocopherol, 500 $\mu \mathrm{M}$ & 0.237 \\
& 0 & & 0.015 \\
& 200 & & 0.045 \\
& 400 & & 0.075 \\
Triton X-100 & 800 & & 0.150 \\
& 800 & Cyclosporin A, $5 \mu \mathrm{M}$ & 0.066 \\
& 800 & $\alpha$-Tocopherol, $500 \mu \mathrm{M}$ & 0.171 \\
& 100 & & 0.069 \\
& 200 & & 0.235 \\
& 200 & Cyclosporin A, $5 \mu \mathrm{M}$ & 0.099 \\
& 200 & $\alpha$-Tocopherol, 500 $\mu \mathrm{M}$ & 0.168 \\
\hline
\end{tabular}

Induction of MMPT measured by swelling of mitochondria, as determined by change in absorbance ( $\Delta \mathrm{OD}$ ) at $540 \mathrm{~nm}$ after the addition of detergent (absorbance at $5 \mathrm{~min}-$ absorbance at $0 \mathrm{~min}$ ). These experiments were conducted using glutamate/malate as the respiratory substrate.

* Values for $\triangle \mathrm{OD}$ are mean of three to four experiments.

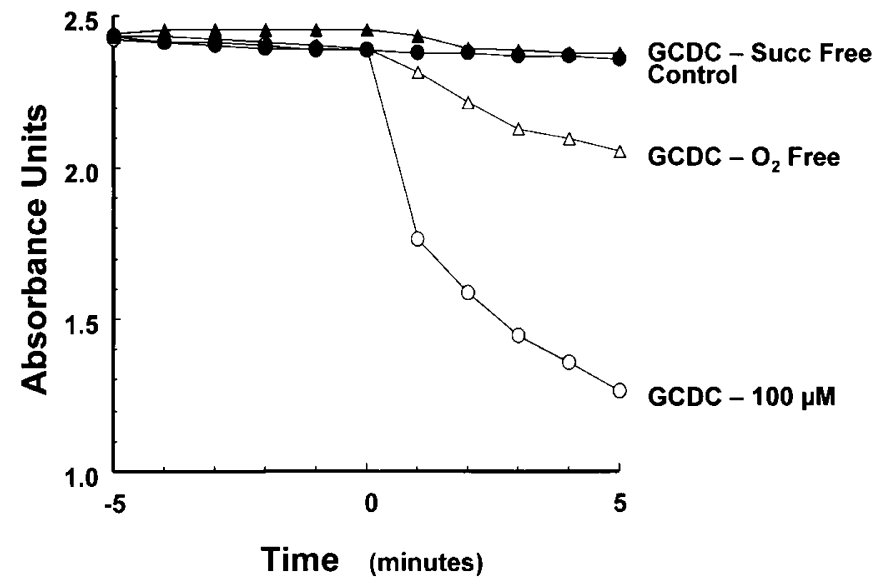

Figure 2. Effect of oxygen-free buffer, and absence of respiratory substrates, on MMPT induced by $100 \mu \mathrm{M}$ GCDC. MMPT experiment was conducted using succinate as the respiratory substrate in buffer under ambient atmosphere (Control and GCDC-100 $\mu M$ ) and in oxygen-free buffer with GCDC (GCDC$\mathrm{O}_{2}$-free), and using no respiratory substrates with GCDC under ambient atmosphere (GCDC-Succ-Free). GCDC induction of the MMPT was dependent on the presence of oxygen and respiratory substrates. Values are mean of three experiments.

and the protocol for the GCDC induction of the MMPT was followed. $\alpha$-Tocopherol inhibited the magnitude of the MMPT $(\Delta \mathrm{OD})$ in a dose-dependent manner during succinate- (Fig. 4) and glutamate/malate- (Table 1) stimulated respiration. In mitochondria exposed to 25 and $50 \mu \mathrm{M}$ GCDC, preincubation with $200 \mu \mathrm{M} \alpha$-tocopherol had a similar effect of reducing MMPT by $50-60 \%$ (data not shown). A series of other antioxidants also significantly inhibited the MMPT when preincubated before exposure of mitochondria to $100 \mu \mathrm{M}$ GCDC (Fig. $5 A$ ). Of particular interest was the suppression of further changes in absorbance when the antioxidants were added after the GCDC had initiated the onset of the MMPT (Fig. 6).

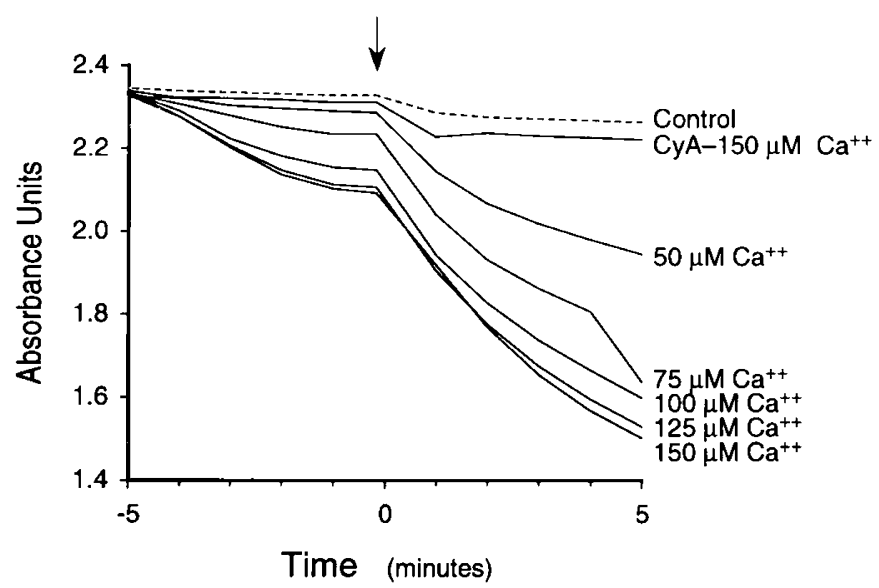

Figure 3. Induction of the MMPT by GCDC $(200 \mu \mathrm{M})$ requires calcium. Absorbance of mitochondrial suspension was monitored at $540 \mathrm{~nm}$ as in Figure $1 B$, except that the concentration of calcium chloride (added at $\mathrm{t}=-5 \mathrm{~min}$ ) was varied between 0 and $150 \mu \mathrm{M}$ and the concentration of GCDC added at $\mathrm{t}=0 \mathrm{~min}$ (vertical arrow) was $200 \mu \mathrm{M}$ for all experiments. Values shown are mean of four experiments.

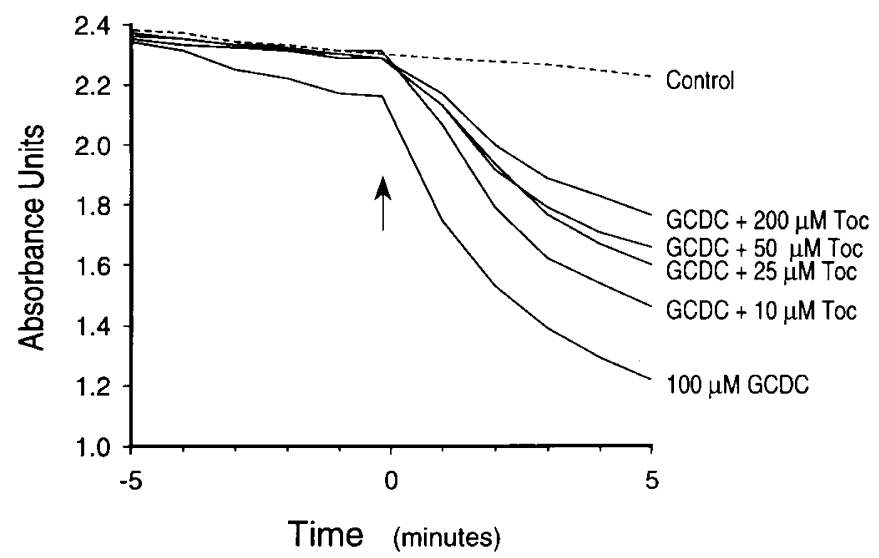

Figure 4. $\alpha$-Tocopherol (Toc) inhibits the MMPT induced by GCDC during succinate-stimulated respiration. MMPT experiment was conducted as in Figure $1 B$ with the following modifications: Mitochondria were preincubated from $\mathrm{t}=-10 \mathrm{~min}$ to $\mathrm{t}=0 \mathrm{~min}$ with $0-200 \mu \mathrm{M} \mathrm{R}, \mathrm{R}, \mathrm{R}-\alpha$-tocopherol dissolved in $0.5 \%$ DMSO, or the volume of $0.5 \%$ DMSO used for the highest concentration of $\alpha$-tocopherol. At $\mathrm{t}=0 \mathrm{~min}, 100 \mu \mathrm{M}$ GCDC was added. $\alpha$-Tocopherol significantly inhibited the MMPT in a concentration-dependent manner. Values shown are the mean of three experiments.

In three experiments, transmission electron microscopy was performed on mitochondria after isolation and before the addition of GCDC and again 5 min after the addition of $100 \mu \mathrm{M}$ GCDC. GCDC induced significant swelling of mitochondria with decreased matrix density and loss of visible cristae (Fig. 7) and an increase in cross-sectional area per mitochondrion (Table 2). This swelling was significantly inhibited by pretreatment of the mitochondria with cyclosporin $\mathrm{A}$ and with the antioxidants $\alpha$-tocopherol and ebselen (Fig. 7, Table 2). These morphologic data confirmed the findings of the spectrophotometric assay for mitochondrial swelling.

Experiments were conducted to determine the time course of ROS generation as related to the MMPT induced by GCDC. Employing the DCFein probe as an indicator of hydroperoxide generation, GCDC stimulated the generation of ROS in a 

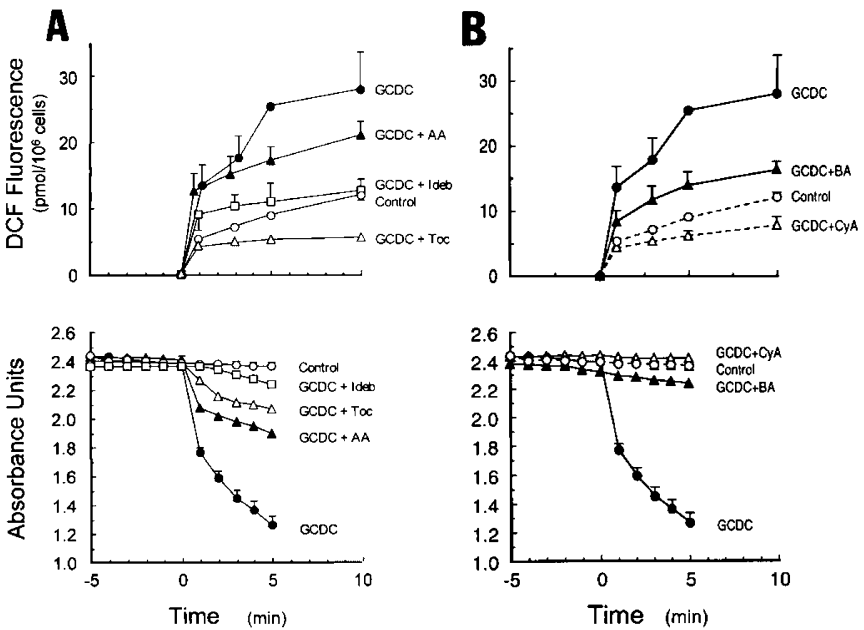

Figure 5. Generation of hydroperoxides (DCF fluorescence) and induction of MMPT (change in absorbance units) in isolated hepatic mitochondria exposed to $100 \mu \mathrm{M}$ GCDC during succinate-driven respiration. $(A)$ In mitochondria exposed to GCDC, hydroperoxide generation paralleled the decrease in absorbance, which represents induction of the MMPT. Treatment of mitochondria with the antioxidants $\alpha$-tocopherol (Toc; $100 \mu \mathrm{M})$, ascorbic acid $(A A ; 1 \mathrm{mM})$, and idebenone $(I d e b ; 10 \mu \mathrm{M})$ suppressed generation of hydroperoxides proportional to degree of inhibition of MMPT. $(B)$ Treatment of mitochondria with MMPT blockers, bongkrekic acid $(B A ; 5 \mu \mathrm{M})$, and cyclosporin A $(C y A$; $1 \mu \mathrm{M})$, reduced the decrease in absorbance and reduced generation of hydroperoxides. Values are mean \pm SEM of four experiments.

concentration-dependent manner and over the same time course $(5 \mathrm{~min})$ as the induction of the MMPT (Fig. 5A). Moreover, preincubation of mitochondria with antioxidants followed by the addition of GCDC led to significantly reduced hydroperoxide generation (Fig. 5A) in parallel to the inhibition of the MMPT. Suppression of the MMPT by preincubation with the MMPT blockers, cyclosporin A, and bongkrekic acid, was unexpectedly associated with significant inhibition of hydroperoxide generation as well as the expected inhibition of mitochondrial swelling (Fig. 5B).

\section{Effect of Detergents on Induction of the MMPT}

One possible mechanism of MMPT induction by bile acids may be related to their detergent action upon mitochondrial membranes, particularly the inner membrane. Therefore, we examined whether two other detergents (44) were capable of inducing the MMPT: CHAPS, which is structurally and biophysically very similar to bile acids, and Triton X-100, which is structurally unrelated to bile acids but somewhat similar in detergent activity.

One index of detergent activity is the critical micellar concentration (CMC). In solutions of $0.1-0.2 \mathrm{M} \mathrm{Na}^{+}$, the CMC of GCDC is $1.0-1.5 \mathrm{mM}$, while that of CHAPS is $3.0-5.0 \mathrm{mM}$ $(44,45)$. Therefore, we reasoned that if the detergent properties of GCDC on the inner mitochondrial membrane were involved in induction of the MMPT, then a concentration of CHAPS that would produce similar or somewhat higher detergent activity (200 $\mu$ M GCDC should be equivalent to approximately $400-$ $500 \mu \mathrm{M}$ CHAPS) should also induce the MMPT. We examined a range of concentrations of CHAPS and observed that even at the highest, $800 \mu \mathrm{M}$, the magnitude of mitochondrial
A

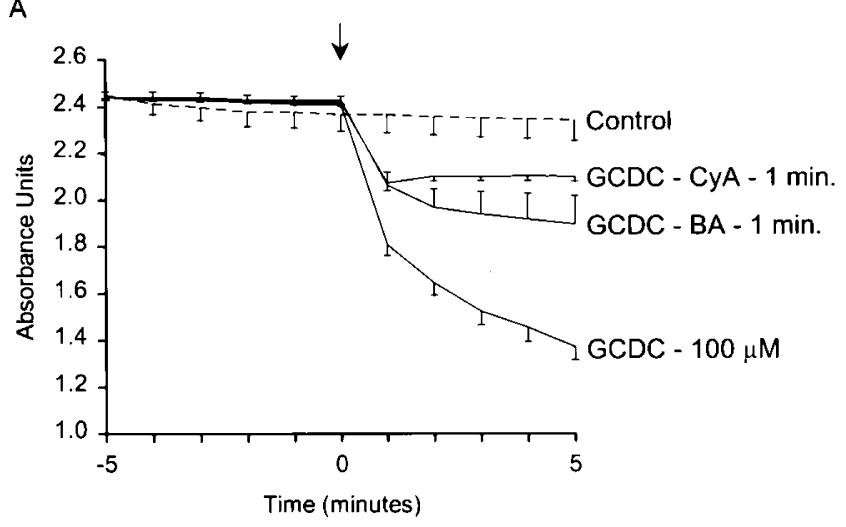

B

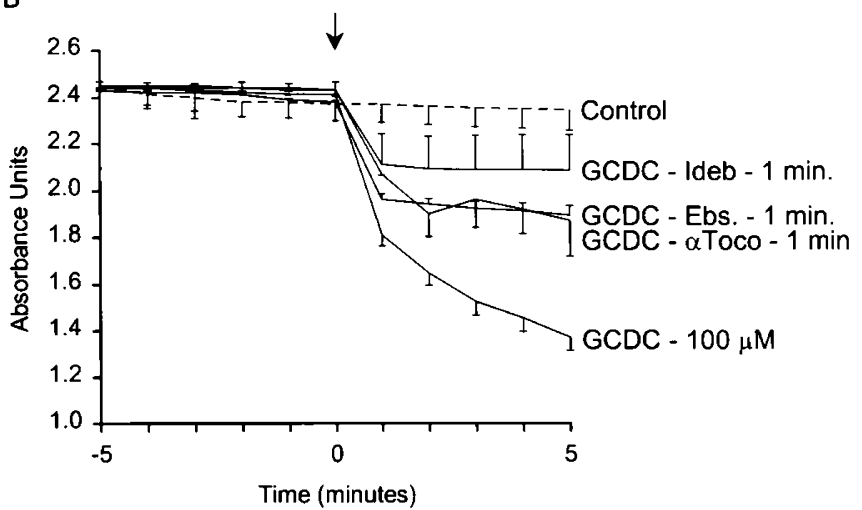

Figure 6. Effect of addition of MMPT blockers $(A)$ and antioxidants $(B)$ after induction of MMPT by GCDC. MMPT was measured as in Figure $1 B$ except that $5 \mu \mathrm{M}$ bongkrekic acid $(B A-1 \mathrm{~min}), 1 \mu \mathrm{M}$ cyclosporin $\mathrm{A}(C y A-1 \mathrm{~min}), 200$ $\mu \mathrm{M} \alpha$-tocopherol ( $\alpha$-Toco-1 min), $25 \mu \mathrm{M}$ ebselen $(E b s-1 \mathrm{~min}$ ), or $10 \mu \mathrm{M}$ idebenone (Ideb-1 $\mathrm{min}$ ) were added $1 \mathrm{~min}$ after the addition of $100 \mu \mathrm{M} \mathrm{GCDC}$ at 0 min. The MMPT blockers and antioxidants prevented further mitochondrial swelling after their addition.

swelling $(\triangle \mathrm{OD})$ was less than one-third of that induced by 200 $\mu \mathrm{M}$ GCDC (Table 1). CHAPS-induced mitochondrial swelling was reduced by $56 \%$ in the absence of $\mathrm{Ca}^{2+}$, by $56 \%$ in the presence of cyclosporin $\mathrm{A}$, and not at all by $\alpha$-tocopherol (Table 1).

The polyoxyethylene detergent Triton X-100 is structurally different from GCDC, and has a CMC of approximately $0.3 \mathrm{mM}$, less than $30 \%$ that of GCDC. Therefore, equivalent detergent activity of Triton X-100 to that of $200 \mu \mathrm{M}$ GCDC would be reached at approximately $60 \mu \mathrm{M}$ Triton X-100. We studied two concentrations of Triton X-100 that exceeded this concentration and found minimal induction of mitochondrial swelling (Table 1). The absence of $\mathrm{Ca}^{2+}$ reduced swelling by $64 \%$, cyclosporin A by $58 \%$ and $\alpha$-tocopherol by $29 \%$ (Table 1). Therefore, Triton X-100, at concentrations resulting in higher detergent activity than $200 \mu \mathrm{M}$ GCDC, produced a relatively small degree of mitochondrial swelling compared with GCDC. The results of these experiments with Triton X-100 and CHAPS implicated a property other than the detergent effect of GCDC in its ability to induce the MMPT. 


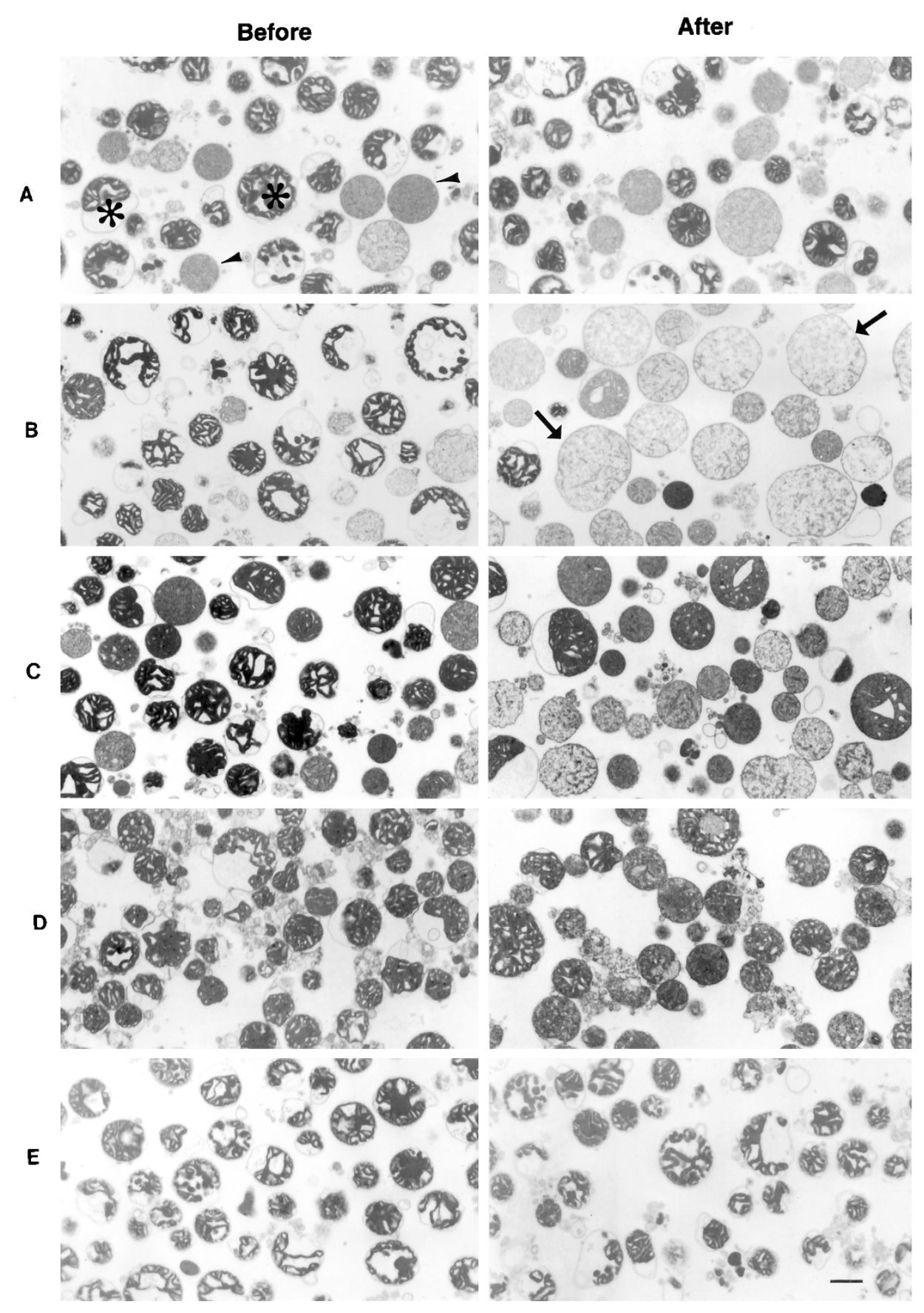

Figure 7. Ultrastructural evidence of prevention of bile acid-induced MMPT by antioxidant treatment. Electron micrographs of mitochondria removed from the MMPT assay immediately before (left column) the addition of $100 \mu \mathrm{M} \mathrm{GCDC}(B-E)$, but after preincubation with $200 \mu \mathrm{M} \alpha$-tocopherol $(C), 25 \mu \mathrm{M}$ ebselen $(D)$, or $1.0 \mu \mathrm{M}$ cyclosporin A $(E)$ as described in the "Methods" section. In the right column are electron micrographs of mitochondria from the same experiments removed 5 min after the addition of GCDC in rows $B-E$. Row $A$ illustrates the morphology of mitochondria incubated under control conditions without GCDC and without the addition of reagents in rows $B-E$. After the preincubation period, the mitochondria (A-Before) are mostly in a condensed state (asterisk) although some have a less dense matrix but are not swollen (arrow heads). After 5 min incubation, the mitochondria (A-After) appear unchanged, whereas GCDC exposure (B-After) caused the majority of mitochondria to swell and develop a dispersed matrix (arrows). The MMPT induced by GCDC was prevented by the antioxidants $\alpha$-tocopherol (C-After) and ebselen (D-After). Cyclosporin A (E-After) prevented the mitochondrial swelling induced by GCDC, confirming that the MMPT is the basis for the mitochondrial swelling. Bar in E-After is $0.5 \mu \mathrm{m}$.

\section{The Calpain Inhibitor, Cbz-Leu-Leu-Tyr, Has No Effect on the GCDC-induced MMPT}

Recently, Aguilar, et al. (46) demonstrated that the calpain protease inhibitor, Cbz-Leu-Leu-Tyr, reduced the magnitude of the MMPT induced by tert-butyl hydroperoxide and by $\mathrm{Ca}^{2+}$, and proposed that mitochondrial calpain activity was a medi- ator of the GCDC-induced MMPT (20). To determine whether calpain proteases were involved in the GCDC-induced MMPT, we examined the effect of the calpain inhibitor, Cbz-Leu-LeuTyr, on MMPT in a manner similar to that reported by Aguilar et al. (46) (Fig. 8). Preincubation of mitochondria for $15 \mathrm{~min}$ with $100 \mu \mathrm{M}$ Cbz-Leu-Leu-Tyr during glutamat/malate -stim- 
Table 2. Quantitative analysis of cross-sectional area of mitochondria

\begin{tabular}{|c|c|c|c|c|}
\hline Time (min) & GCDC $(\mu \mathrm{M})$ & $\begin{array}{l}\text { Cyclosporin A } \\
\text { concentration }\end{array}$ & $\begin{array}{c}\text { Antioxidant } \\
\text { (concentration) }\end{array}$ & $\begin{array}{c}\text { Cross-sectional area } \\
\left(\mu \mathrm{m}^{2} \text { per mitochondrion }\right)\end{array}$ \\
\hline 0 & 0 & 0 & & 0.59 \\
\hline 5 & 0 & 0 & & 0.68 \\
\hline 5 & $100 \mu \mathrm{M}$ & 0 & & 1.67 \\
\hline 0 & $100 \mu \mathrm{M}$ & $5 \mu \mathrm{M}$ & & 0.49 \\
\hline 5 & $100 \mu \mathrm{M}$ & $5 \mu \mathrm{M}$ & & 0.48 \\
\hline 0 & $100 \mu \mathrm{M}$ & 0 & Ebselen $(25 \mu \mathrm{M})$ & 0.44 \\
\hline 5 & $100 \mu \mathrm{M}$ & 0 & Ebselen $(25 \mu \mathrm{M})$ & 0.56 \\
\hline
\end{tabular}

Cross-sectional area of isolated hepatic mitochondria was determined on electron micrographs by electronic scanning techniques before $(0$ min) and after ( 5 min) addition of 0 or $100 \mu \mathrm{M} \mathrm{GCDC}$, with or without pretreatment with the MMPT blocker, cyclosporin A, or the antioxidants, $\alpha$-tocopherol or ebselen. For details of experiments, see "Methods." At least 100 mitochondria were evaluated for each experimental condition. Values are expressed at the mean of three experiments.

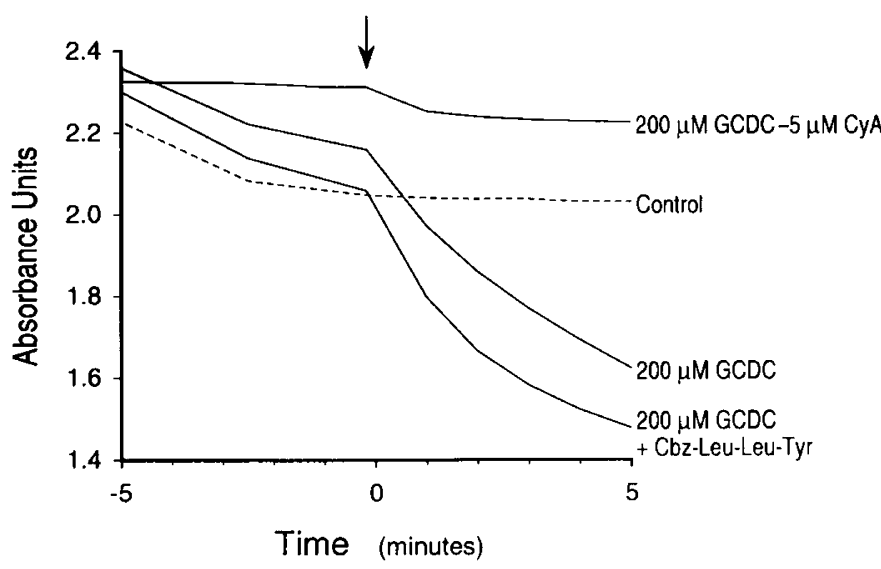

Figure 8. The protease inhibitor Cbz-Leu-Leu-Tyr had no effect on the MMPT induced by GCDC. Absorbance of mitochondrial suspension was monitored at $540 \mathrm{~nm}$ as in Figure $1 A$ with the following changes: 0 or $200 \mu \mathrm{M}$ GCDC was added at $\mathrm{t}=0$ min (vertical arrow) to buffer containing $100 \mu \mathrm{M}$ calcium chloride and $1 \mathrm{mM}$ glutamate/malate that was preincubated from $\mathrm{t}=$ $-20 \mathrm{~min}$ to $\mathrm{t}=-5 \mathrm{~min}$ with 0 or $100 \mu \mathrm{M}$ of the protease inhibitor, Cbz-Leu-Leu-Tyr, or to buffer preincubated from $t=-15 \min$ to $t=-5 \mathrm{~min}$ with $5 \mu \mathrm{M}$ cyclosporin A dissolved in ethanol. Values shown are the mean of three experiments.

ulated respiration had no effect on the magnitude of the MMPT

\section{ROS Generation by Mitochondria is Independent of MMPT Induction}

The unexpected findings that cyclosporin A led to a reduction of ROS generation in mitochondria (Fig. 5B) and hepatocytes (see below) exposed to GCDC raised the question of whether opening of the permeability pore was itself responsible for the ROS generation in bile acid toxicity. To address this issue, isolated mitochondria were preincubated with cyclosporin A in decreasing concentrations and then the MMPT was induced with $100 \mu \mathrm{M}$ GCDC as described above. As cyclosporin A concentrations were reduced, a clear threshold was reached in four separate experiments, below which there was no inhibition of the GCDC-induced MMPT (Fig. 9). However, generation of ROS measured by DCFein fluorescence did not change significantly at concentrations of cyclosporin A directly above or below this threshold (Fig. 9), although at higher

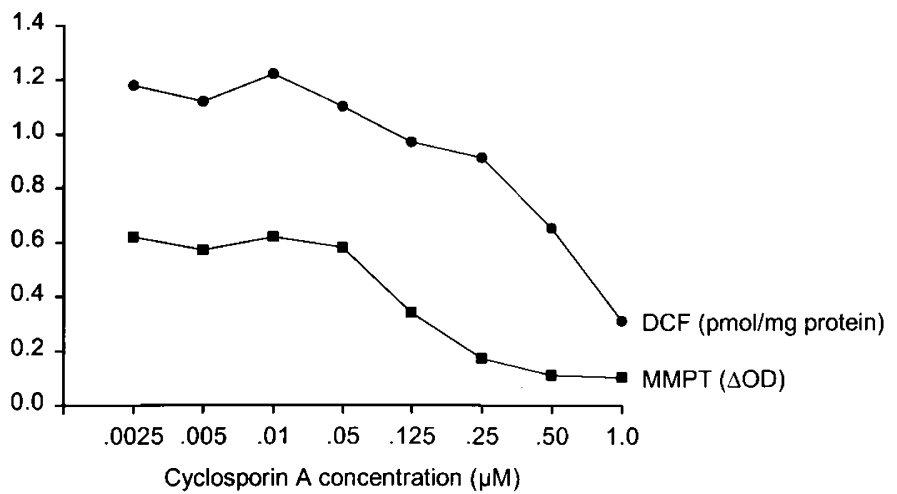

Figure 9. Comparison of decrease of absorbance units representing MMPT induction $(\triangle \mathrm{OD})$ with generation of hydroperoxides (DCF fluorescence) in isolated hepatic mitochondria exposed for $5 \mathrm{~min}$ to $100 \mu \mathrm{M}$ GCDC. Mitochondria were preincubated for $10 \mathrm{~min}$ with varying concentrations of cyclosporin A and then the MMPT was stimulated by GCDC for $5 \mathrm{~min}$ as in Figure $1 B$. Generation of hydroperoxides (measured 5 min after addition of GCDC) did not change significantly at concentrations of cyclosporin A ( 0.25 and 0.05 $\mu \mathrm{M})$ that were above and below the cyclosporin A threshold that inhibited the MMPT $(0.125 \mu \mathrm{M})$, indicating that opening of the permeability pore was not responsible for the generation of hydroperoxides. Data are shown for one experiment representative of four experiments performed.

concentrations of cyclosporin A there was a mild inhibition of DCFein fluorescence. Thus, GCDC-induced generation of ROS by hepatic mitochondria was not dependent on the induction of the MMPT.

\section{Blocking the MMPT Protects Hepatocytes and Reduces Oxidant Stress During GCDC Exposure}

Having demonstrated that antioxidants inhibited the GCDCinduced MMPT in mitochondria and with prior studies showing that antioxidants protected hepatocytes against bile acidinduced necrosis $(17,18)$, we next determined if GCDC induced the MMPT in isolated hepatocytes and if blocking the MMPT protected hepatocytes from bile acid-induced necrosis. We first used flow cytometry (Fig. 10) to determine whether GCDC induced the MMPT in freshly isolated rat hepatocytes before the onset of cellular necrosis. Using the fluorescent dyes, JC-1 that aggregates in the presence of mitochondrial $\Delta \psi$ and TMRM which accumulates in mitochondria proportional 
to the membrane potential, we demonstrated a loss of $\Delta \psi$ in isolated hepatocytes after $1 \mathrm{~h}$ exposure to $500 \mu \mathrm{M}$ GCDC (Fig. 10). JC-1 monomer fluorescence was similar in cells before and after exposure for $1 \mathrm{~h}$ to 0 or $500 \mu \mathrm{M}$ GCDC, confirming similar uptake of JC-1 into hepatocytes. Therefore, the decreased JC-1 aggregate fluorescence and TMRM fluorescence (that was inhibited by cyclosporin A) represented a reduction in $\Delta \psi$, and hence, by inference, induction of the MMPT. These data were similar to that obtained when isolated hepatocytes were exposed to valinomycin or FCCP, two compounds that dissipate the mitochondrial $\Delta \psi$ (data not shown). Preincubation of hepatocytes for $15 \mathrm{~min}$ with $\alpha$-tocopherol $(250 \mu \mathrm{M})$ or idebenone $(100 \mu \mathrm{M})$ before addition of $500 \mu \mathrm{M}$ GCDC prevented loss of $\Delta \psi$ (Fig. 10B, right column), as did preincuabtion with cyclosporin A $(5 \mu \mathrm{M})$. Thus, $500 \mu \mathrm{M}$ GCDC not only induced the MMPT in isolated hepatocytes (indicated by loss of $\Delta \psi$ that was prevented by cyclosporin A), but antioxidants significantly attenuated the MMPT magnitude.

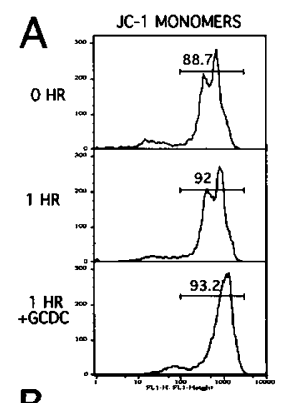

B

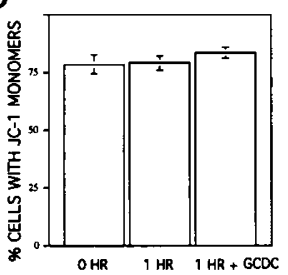

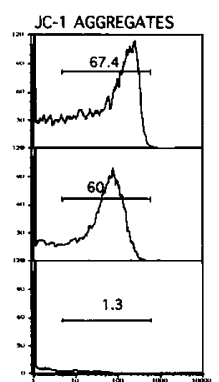

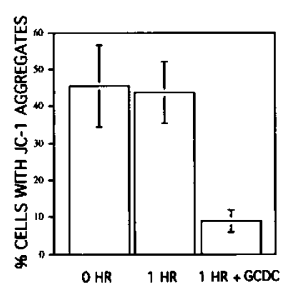

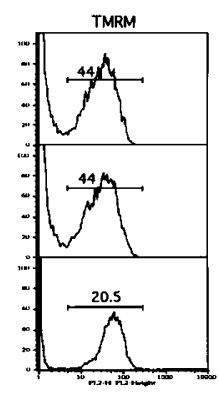

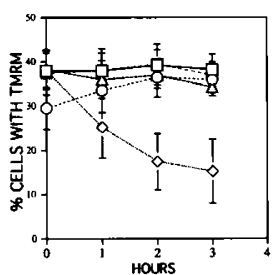

Figure 10. FACS analysis of fluorescent probes, JC-1 and TMRM, in isolated rat hepatocytes incubated with $500 \mu \mathrm{M}$ GCDC for up to $4 \mathrm{~h}$. (A) Histograms from two representative experiments using JC-1 (left and middle columns) and TMRM (right column) indicate that exposure to $500 \mu \mathrm{M}$ GCDC for $1 \mathrm{~h}$ caused a significant collapse of mitochondrial membrane $\Delta \psi$. On the histograms, $y$ axis indicates cell number, $x$ axis the log of fluorescence intensity (four orders of magnitude are displayed), and the percentage of cells (number above the bar) having the fluorescence intensity indicated under the bar. Top histogram in each column (0 HR) represents control cells before incubation with GCDC, middle histogram (1 HR) represents nontreated cells incubated for $1 \mathrm{~h}$, and the bottom histogram (1HR + GCDC) represents cells incubated for $1 \mathrm{~h}$ with 500 $\mu \mathrm{M}$ GCDC. The left column, JC-1 monomers, indicates that cell loading of the JC-1 was not affected by incubating the cells for $1 \mathrm{~h}$ with or without GCDC. The middle column shows that JC-1 aggregate formation was markedly decreased upon exposure of cells to GCDC, indicating a loss of mitochondrial membrane $\Delta \psi$. In the right column, TMRM data shows a similar collapse of $\Delta \psi$ upon exposure of cells to GCDC for $1 \mathrm{~h}$. (B) Numerical data (mean \pm SEM) summarizing three experiments. Graphs on the left and middle show results of JC-1 monomers and aggregates. Graph on right shows time course for TMRM fluorescence of hepatocytes exposed to $500 \mu \mathrm{M} \mathrm{GCDC} \mathrm{(diamonds)}$ compared with control cells (squares). In cells exposed to GCDC, the MMPT inhibitor cyclosporin A (5 $\mu \mathrm{M}$, circles $)$, and the antioxidants $\alpha$-tocopherol $(250 \mu \mathrm{M}$, triangles) and idebenone $(100 \mu \mathrm{M}$, cross-hatched squares, partially obscured by control symbols), prevented the GCDC-induced collapse of the mitochondrial $\Delta \psi$.
We next sought to determine whether blocking the MMPT protected isolated hepatocytes from bile acid-induced cellular necrosis. Exposure of isolated rat hepatocytes in suspension to $500 \mu \mathrm{M}$ GCDC for $4 \mathrm{~h}$ led to time-dependent increases in LDH release, TBARS generation and DCFein fluorescence (Fig. 11). Preincubation of hepatocytes with the MMPT blockers, cyclosporin A and TFP, significantly reduced LDH release and TBARS generation caused by GCDC exposure (Fig. 11). Treatment with the MMPT blockers also led to approximately a $50 \%$ reduction in DCF fluorescence induced by GCDC. To determine whether this effect of the MMPT blockers was caused by altered hepatocyte uptake or release of GCDC, hepatocyte concentrations of total CDC (conjugated + unconjugated species) were measured throughout these experiments. As expected, cellular concentrations of total CDC increased approximately 10 to 15 -fold after $1 \mathrm{~h}$ of incubation and remained elevated throughout the duration of the experiment (Fig. 12), however, there was no effect of preincubation with cyclosporin A and TFP on the concentrations of cellular total
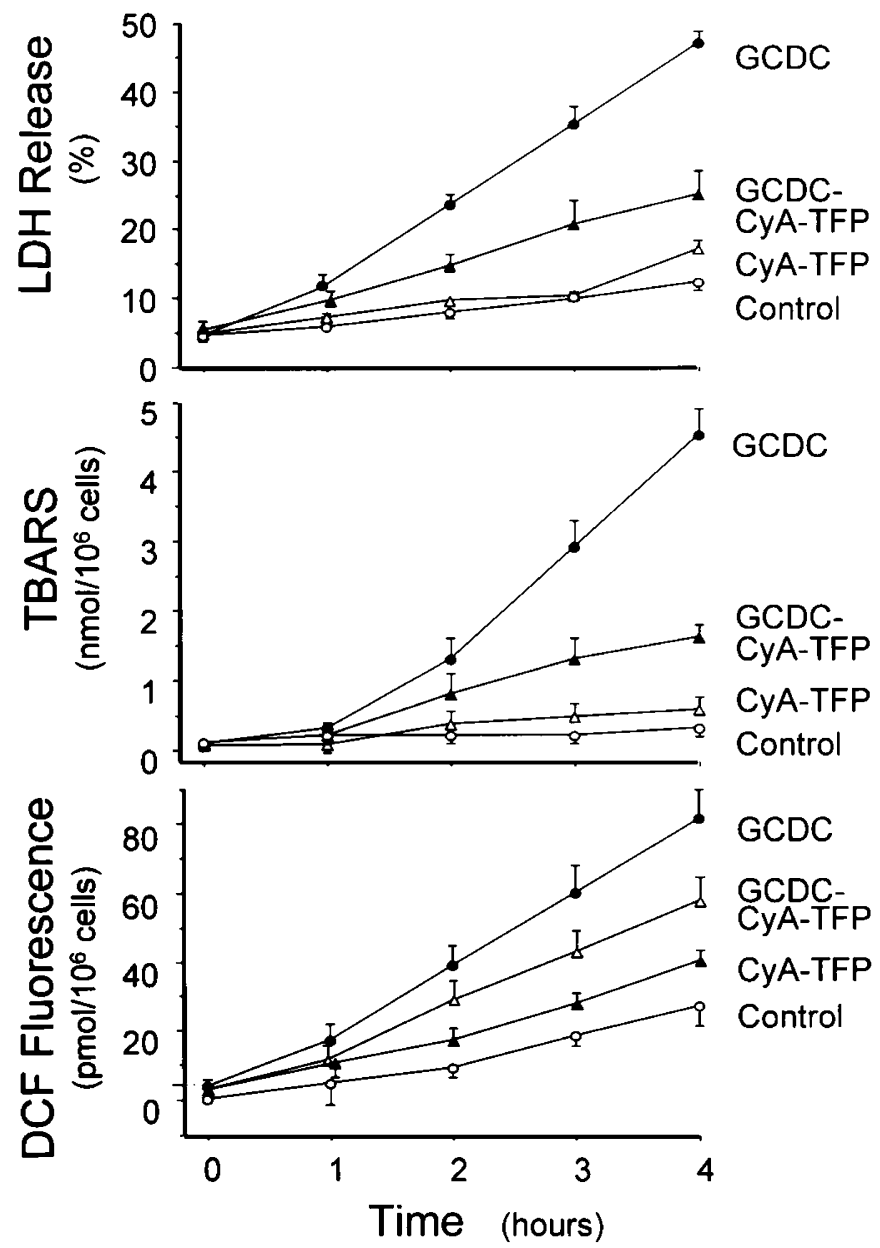

Figure 11. Time course of percentage release of LDH (top), concentrations of thiobarbituric acid reacting substances (TBARS) (middle), and DCF fluorescence (bottom) during exposure of isolated rat hepatocytes to 500 $\mu \mathrm{M}$ GCDC (see text for details of methods). Treatment of cells with inhibitors of the MMPT, cyclosporin A $(C y A ; 5 \mu \mathrm{M})$ and trifluoperazine $(T F P ; 10 \mu \mathrm{M})$, significantly $(p<0.05)$ reduced LDH release and TBARS generation and reduced DCF fluorescence by approximately $50 \%$. Values are mean \pm SEM of three experiments. 


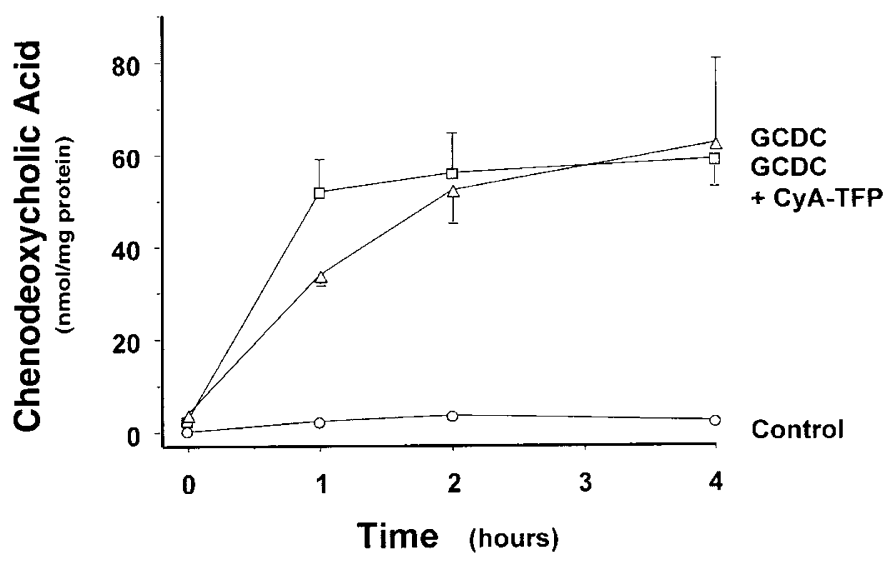

Figure 12. Concentrations of total chenodeoxycholic acid (conjugated + unconjugated $\mathrm{CDC}$ ) in isolated rat hepatocytes exposed to $500 \mu \mathrm{M}$ GCDC over $4 \mathrm{~h}$. CDC concentrations were measured by gas chromatography-mass spectrometry as detailed in "Methods." Treatment of cells with $5 \mu \mathrm{M}$ cyclosporin $\mathrm{A}(C y A)$ and $10 \mu \mathrm{M}$ trifluoperazine $(T F P)$ did not reduce hepatocyte concentrations of CDC throughout the course of the experiment. Values are mean $\pm \mathrm{SEM}$ of three experiments.

CDC at any time point. These elevations of hepatocellular bile acids are in the order of the same magnitude as the increase in hepatic bile acids measured in liver from humans with cholestatic liver disease (1). Thus, the protection against hepatocyte injury and oxidant stress demonstrated by these MMPT blockers was not attributable to effects on GCDC uptake or efflux by hepatocytes.

\section{DISCUSSION}

In this study, we have explored the role that oxidative stress plays in the induction of the MMPT and hepatocyte necrosis caused by hydrophobic bile acids. The results of this study indicate that the hydrophobic bile acid, GCDC, induces the MMPT in isolated hepatic mitochondria at concentrations of bile acids (25-200 $\mu \mathrm{M})$ comparable to those that have been measured in human cholestatic liver (1) and to which mitochondria would be expected to be exposed within the cholestatic hepatocyte (4). Moreover, GCDC stimulates the generation of ROS within minutes in isolated hepatic mitochondria and in intact hepatocytes, coincident with the induction of the MMPT. In mitochondria, both the MMPT and the generation of ROS are significantly attenuated by a variety of antioxidants. Furthermore, detergent properties of bile acids are not responsible for induction of the MMPT, however, the concentration of $\mathrm{Ca}^{2+}$ plays an important role in the GCDC-induced MMPT, as it does in the action of other inducers of the MMPT $(22,23)$. Finally, in freshly isolated rat hepatocytes, GCDC induces the MMPT and hepatocellular necrosis, antioxidants inhibit the MMPT and blockers of the MMPT provide strong protection against cellular necrosis caused by exposure to GCDC. The bile acid concentrations achieved in these hepatocytes exposed to $500 \mu \mathrm{M}$ GCDC for $4 \mathrm{~h}$ are in the range of liver from cholestatic humans and rats (1). Taken together, these data demonstrate that hydrophobic bile acids cause hepatocyte necrosis by a mechanism involving induction of the MMPT and also implicate the generation of ROS within mitochondria as a promoter of the MMPT in this model.
The mechanism by which GCDC stimulates generation of ROS in hepatocytes (17) and mitochondria (47) has not been fully established. Krahenbuhl et al. (19) have shown that hydrophobic bile acids impair state 3 respiration and interrupt normal electron transport at complex III of the respiratory chain, a process that can increase generation of ROS by mitochondria. Preliminary data indicate that electron transfer to oxygen from the ubquinone-complex III interaction in the mitochondrial inner membrane leads to generation of ROS in mitochondria exposed to GCDC (47). The resultant oxidant stress may then lead to depletion of mitochondrial antioxidants or directly oxidize vicinal thiol groups in the permeability pore protein, leading to pore opening (48). The significant attenuation of the MMPT by $\alpha$-tocopherol and idebenone, two membrane-associated antioxidants, and less so by ascorbate that does not associate with membranes, is in support of this proposed site of ROS generation. The requirement for $\mathrm{Ca}^{2+}$ suggests that $\mathrm{Ca}^{2+}$ binding to the pore is also necessary for the GCDC-induced MMPT, and that oxidant stress caused by GCDC is necessary, but not sufficient, for pore opening to occur in this model. Other mechanisms have been proposed for the opening of the permeability pore by ROS and other prooxidants (e.g. t-butyl hydroperoxide, Fe(II)citrate, xanthine oxidase, and thiol-oxidizing agents) (22, 23, 49-51). MMPT induced by pro-oxidants may be triggered by $\mathrm{Ca}^{2+}$-stimulated production of ROS (51-53), perhaps by increasing mitochondrial respiration that is under the control of the activity of mitochondrial dehydrogenases that are regulated by mitochondrial matrix free $\mathrm{Ca}^{2+}$ (54). Alternatively, pro-oxidants may deplete mitochondrial antioxidant defenses (glutathione and NADPH), which favors the accumulation of mitochondrial ROS generated by the effect of $\mathrm{Ca}^{2+}$ on the respiratory chain, resulting in oxidation of critical thiol groups in the membrane pore and its opening (52). Finally, it has also been proposed that oxidative stress causes release of matrix $\mathrm{Ca}^{2+}$ that can be taken up again by the mitochondria $\left(\mathrm{Ca}^{2+}\right.$ cycling) and that the excessive $\mathrm{Ca}^{2+}$ cycling may be responsible for the MMPT (50). The dependence on $\mathrm{Ca}^{2+}$ of the GCDC-induced MMPT is consistent with either of these mechanisms.

In other cellular systems, it has been proposed that opening of the mitochondrial permeability pore itself stimulates generation of ROS by interruption of normal electron transport (55) caused by the release of mitochondrial cytochrome $c$ to cytosol (56). In our studies, chemical blockade of the MMPT in hepatocytes exposed to GCDC resulted in a modest reduction of hydroperoxide generation in association with protection against necrosis (Fig. 11). In addition, ROS generation in isolated mitochondria paralleled the opening of the MMPT (Fig. 5). We further addressed this issue by performing a dose-response experiment in isolated mitochondria and observed that ROS generation did not vary significantly at low concentrations of cyclosporin A near the threshold for inhibition of the MMPT (Fig. 9). Higher concentrations of cyclosporin A had a mild inhibitory effect on ROS generation, possibly through direct inhibition of mitochondrial respiration (57). Thus, there was clearly a component of the ROS generated that was not dependent on opening of the permeability pore, although pore opening may have generated ROS to some 
extent, as well. In other models, stimulation of the MMPT promotes ROS generation by mitochondria, possibly through the release of cytochrome $c(56)$. In mitochondria depleted in cytochrome $c$, the respiratory chain protein complexes upstream of cytochrome $c$ become highly reduced and directly transfer their electrons to oxygen, forming superoxide (56). It has been proposed that this autocatalytic mechanism might explain the synchronization of MMPT onset that has been observed by confocal microscopy in single hepatocytes after exposure to tert-butyl hydroperoxide (26).

We determined whether the detergent properties of GCDC on mitochondrial membrane structure could account for the induction of the MMPT in our model system by testing two other detergents. Inasmuch as hydrophobic bile acids are capable of solubilizing both membrane lipids and proteins, we chose a detergent (CHAPS) that would solubilize primarily membrane lipids and another (Triton X-100) that would solubilize both membrane lipids and proteins (44). Neither detergent induced significant high-amplitude mitochondrial swelling at concentrations equivalent to $200 \mu \mathrm{M}$ GCDC (based on relative $\mathrm{CMC}$ equivalency), and did so minimally at higher concentrations, suggesting that the detergent action of GCDC on the mitochondrial membrane plays a minor role, if any, in the induction of the MMPT.

Aguilar et al. (46) recently reported the presence of calpainlike protease activity in hepatocyte mitochondria, which seemed to be involved in the MMPT induced by $\mathrm{Ca}^{2+}$ and by $t$-butyl hydroperoxide, an agent that produces oxidative stress and hepatocyte necrosis $(46,58)$. These investigators demonstrated that the cysteine protease inhibitor, Cbz-Leu-Leu-Tyr (100 $\mu \mathrm{M})$, inhibited mitochondrial high-amplitude swelling induced by both $100 \mu \mathrm{M} \mathrm{Ca}^{2+}$ and by $50 \mu \mathrm{M} t$-butyl hydroperoxide, and that preincubation with Cbz-Leu-Leu-Tyr delayed the loss of the mitochondrial membrane potential and the onset of hepatocyte necrosis caused by $t$-butyl hydroperoxide (46). To determine whether similar calpain-like activity played a role in the GCDC-induced MMPT observed in our experiments, we preincubated mitochondria for 15 min with CbzLeu-Leu-Tyr and then exposed the mitochondria to $200 \mu \mathrm{M}$ GCDC in the presence of $100 \mu \mathrm{M} \mathrm{Ca}^{2+}$. Contrary to the observations of Aguilar et al. (46), we observed no effect of the protease inhibitor on the MMPT in our model (Fig. 8). Thus, it is unlikely that mitochondrial calpain-like protease activity plays a major role in the MMPT induced by GCDC.

It is recognized that hepatocellular necrosis is not the only mechanism of cell death induced by toxic bile acids. Patel et al. (7) have reported that low concentrations of hydrophobic bile acids can induce apoptosis in primary cultured rat hepatocytes. The Fas signaling (36) and protein kinase C pathways (35) have been implicated in bile acid-induced apoptosis. In apoptosis, it is possible that direct activation of Fas by bile acids (36) causes caspase 8 activation that truncates bid, a member of the bcl-2 family, that translocates to mitochondria where it may open the permeability pore by unknown mechanisms, inducing ROS generation (59). Recent studies have suggested that the increased activation of Fas by bile acids may be mediated by the promotion of cytoplasmic transport of Fas to the cell surface by a Golgi- and microtubule-dependent pathway (60).
Preliminary data from our laboratory demonstrate involvement of ROS generation and induction of the MMPT during bile acid-induced apoptosis $(61,62)$. The beneficial effects of ursodeoxycholic acid, a hydrophilic bile acid, in reducing hepatocyte apoptosis may also be related to reduction of ROS generation in mitochondria and inhibition of the MMPT (37). However, Benz et al. (63) recently provided evidence that the Fas receptor pathway may not be involved in apoptosis stimulated by GCDC in human hepatocytes in primary culture. Thus, elucidating the relationships between oxidant stress and the activation of the Fas receptor, bid and bax translocation to the mitochondria, induction of the MMPT, cytochrome $c$ release from mitochondria, and activation of caspases in bile acid-induced apoptosis requires further investigation.

In conclusion, in this study we have demonstrated that concentrations of GCDC representative of those that accumulate in the cholestatic liver $(1,4)$ induce the MMPT in hepatic mitochondria by a mechanism dependent on the generation of ROS and the presence of $\mathrm{Ca}^{2+}$. Because antioxidants have previously been shown to prevent hepatocellular necrosis and reduce oxidant stress in isolated hepatocytes exposed to hydrophobic bile acids $(17,18)$, and, in the current study, to inhibit dissipation of mitochondrial $\Delta \psi$, it is postulated that induction of the MMPT by ROS generated in hepatocyte mitochondria $(19,47)$ is a critical event promoting bile acidinduced hepatocyte necrosis. Elevation of the cytosolic free $\mathrm{Ca}^{2+}$ concentration induced by hydrophobic bile acids (64) may also be an important permissive factor that allows the oxidant stress to open the permeability pore. Thus, novel approaches to reduce the generation of mitochondrial-derived ROS or to prevent increases in mitochondrial $\mathrm{Ca}^{2+}$ concentration may have a beneficial effect in human liver diseases associated with the accumulation of hydrophobic bile acids. The concentrations of bile acids achieved in the isolated hepatocyte experiments and used in the mitochondrial experiments in this study are in the range of those measured in liver from humans with cholestasis $(1,65)$, making the findings reported of potential clinical relevance. The inhibition of the MMPT demonstrated in our study when antioxidants were added after mitochondrial exposure to GCDC suggests that this therapeutic strategy could be of potential benefit, even after the onset of cholestasis and the hepatic accumulation of bile acids. The relative contribution of cellular necrosis versus apoptosis to liver injury in clinical cholestasis has yet to be determined, however, prevention of oxidant stress and inhibition of the MMPT may be possible strategies to reduce both kinds of cellular injury in cholestasis.

\section{REFERENCES}

1. Greim H, Czygan P, Schaffner F, Popper H 1973 Determination of bile acids in needle biopsies of human liver. Biochem Med 8:280-286

2. Attili F, Angelico M, Cantafora A, Alvaro D, Capocaccia L 1986 Bile acid-induced liver toxicity: relation to the hydophobic-hydrophilic balance of bile acids. Med Hypothesis 19:57-69

3. Armstrong MJ, Carey MC 1982 The hydrophobic-hydrophilic balance of bile salts. Inverse correlation between reverse-phase high performance liquid chromatographic mobilities and micellar cholesterol-solubilizing capacities. J Lipid Res 23:70-80

4. Spivey JR, Bronk SG, Gores GJ 1993 Glycochenodeoxycholate-induced lethal cell injury in rat hepatocytes. J Clin Invest 92:17-24

5. Galle PR, Theilmann L, Raedsch R, Otto G Stiehl A 1990 Ursodeoxycholate reduces hepatotoxicity of bile salts in primary human hepatocytes. Hepatology 12:486-491 
6. Sokol RJ, McKim Jr JM, Goff MC, Devereaux MW, Ruyle SZ, Han D, Packer L, Everson G 1998 Vitamin E reduces oxidant injury to mitochondria and hepatotoxicity of intravenous taurochenodeoxycholic acid in the rat. Gastroenterology 114:164-174

7. Patel T, Bronk SF, Gores GJ 1994 Increases of intracellular magnesium promote glycodeoxycholate-induced apoptosis in rat hepatocytes. J Clin Invest 94:2183-2192

8. Rosser BG, Gores GJ 1995 Liver cell necrosis: cellular mechanisms and clinical implications. Gastroenterology 108:252-275

9. Gores GJ, Herman B, Lemasters JJ 1990 Plasma membrane bleb formation and rupture: a common feature of hepatocellular injury. Hepatology 11:690-698.

10. Phillips MJ 1994 Mechanisms and morphology of cholestasis. In: Suchy FJ (ed). Liver Disease in Children. CV Mosby, St. Louis, pp 129-144

11. Scheuer PJ 1980 Liver Biopsy Interpretation. Bailliere Tindall, London, pp 36-59

12. Dahm LJ, Hewett JA, Roth RA 1988 Bile and bile salts potentiate superoxide anion release from activated rat peritoneal neutrophils. Toxicol Appl Pharmacol 95:82-92

13. Togashi H, Shinzawa H, Wakabayoshi H, Nakamura T, Yamada N, Takahashi T, Ishikawa M 1990 Activities of free oxygen radical scavenger enzymes in human liver. J Hepatology 11:200-205

14. Iritani N, Fukuda E, Kitamura Y 1980 Effect of corn oil feeding on lipid peroxidation in rats. J Nutr 110:924-930

15. Lemonnier F, Cresteil D, Feueant M, Couturier M, Bernard O, Alagille D 1987 Plasma lipid peroxides in cholestatic children. Acta Paediatr Scand 76:928-934

16. Sokol RJ, Devereaux M, Khandwala RA 1991 Effect of dietary lipid and vitamin E on mitochondrial lipid peroxidation and hepatic injury in the bile duct-ligated rat. J Lipid Res 32:1349-1357

17. Sokol RJ, Devereaux M, Khandwala R, O’Brien K 1993 Evidence for involvement of oxygen free radicals in bile acid toxicity to isolated rat hepatocytes. Hepatology 17:869-881

18. Sokol RJ, Winklhofer-Roob BM, Devereaux MW, McKim Jr JM 1995 Generation of hydroperoxides in isolated rat hepatocytes and hepatic mitochondria exposed to hydrophobic bile acids. Gastroenterology 109:1249-1256

19. Krähenbühl S, Talos C, Fischer S, Reichen J 1994 Toxicity of bile acids on the electron transport chain of isolated rat liver mitochondria. Hepatology 19:471-479

20. Gores GJ, Miyoshi H, Botla R, Aguilar HI, Bronk SF 1998 Induction of the mitochondrial permeability transition as a mechanism of liver injury during cholestasis: a potential role for mitochondrial proteases. Bioch Biophys Acta 1366:167-175

21. Botla R, Spivey JR, Aguilar H, Bronk SF, Gores GJ 1995 Ursodeoxycholate (UDCA) inhibits the mitochondrial membrane permeability transition induced by glycochenodeoxycholate: a mechanism of UDCA cytoprotection. J Pharmacol Exp The 272:930-938

22. Gunter TE, Pfeiffer DR 1990 Mechanisms by which mitochondria transport calcium Am J Physiol 258:C755-C786

23. Zoratti M, Szabo I 1995 The mitochondrial permeability transition. Biochim Biophys Acta 1241:139-176

24. Bernardi P, Vassanelli S, Veronese P, Colonna R, Szabo I, Zoratti M 1992 Modulation of the mitochondrial permeability transition pore. J Biol Chem 267:2934-2939

25. Pastorino JG, Snyder JW, Serroni A, Hoek JB, Farber JL 1993 Cyclosporin and carnitine prevent the anoxic death of cultured hepatocytes by inhibiting the mitochondrial permeability transition. J Biol Chem 268:13791-13798

26. Nieminen A-L, Byrne AM, Herman B, Lemasters JJ 1997 Mitochondrial permeability transition in hepatocytes induced by $\mathrm{t}-\mathrm{BuOOH}$ : $\mathrm{NAD}(\mathrm{P}) \mathrm{H}$ and reactive oxygen species. Am J Physiol 272:C1286-C1294

27. Lemasters JJ, Nieminen AL, Qian T, Trost LC, Elmore SP, Nishimura Y, Crowe RA, Cascio WE, Bradham CA, Brenner DA, Herman B 1998 The mitochondrial permeability transition in cell death: a common mechanism in necrosis, apoptosis and autophagy. Biochim Biophys Acta 1366:177-196

28. Susin SA, Zamzami N, Kroemer G 1998 Mitochondria as regulators of apoptosis: doubt no more. Biochim Biophys Acta 1366:151-165

29. Szabo I, Zoratti M 1993 The mitochondrial permeability transition pore may comprise VDAC molecules I: binary structure and voltage dependence of the pore. FEBS Lett 330:201-205

30. Szabo I, De Pinto V, Zoratti M 1993 The mitochondrial permeability transition pore may comprise VDAC molecules II: the electrophysiological properties of VDAC are compatible with those of the mitochondrial megachannel. FEBS Lett 330:206-210

31. Brustovetsky N, Klingenberg M 1996 Mitochondrial ADP/ATP carrier can be reversibly converted into a large channel by $\mathrm{Ca}^{2+}$. Biochemistry 35:8483-8488

32. Woodfield K, Ruck A, Brdiczka D, Halestrap AP 1998 Direct demonstration of specific interaction between cyclophilin-D and the adenine nucleotide translocase confirms their role in the mitochondrial permeability transition. Biochem 336:287-90

33. Fournier N, Ducet G, Crevat A 1987 Action of cyclosporin on mitochondrial calcium fluxes. J Bioenerg Biomembr 19:297-303

34. Broekemeier KM, Dempsey ME, Pfeiffer DR 1989 Cylcosporin A is a potent inhibito of the inner membrane permeability transition in liver mitochondria. J Biol Chem 264:7826-7830

35. Jones BA, Rao Y-P, Stravitz T, Gores GJ 1997 Bile salt-induced apoptosis of hepatocytes involves activation of protein kinase C. Am J Physiol 272:G1109-G1115

36. Faubion WA, Guicciardi ME, Miyoshi H, Bronk SF, Roberts PJ, Svingen PA, Kaufmann SH, Gores GJ 1999 Toxic bile salts induce rodent hepatocyte apoptosis via direct activation of Fas. J Clin Invest 103:137-145

37. Rodrigues CM, Fan G, Wong PY, Kren BT, Steer CJ 1998 Ursodeoxycholic acid may inhibit deoxycholic acid-induced apoptosis by modulating mitochondrial transmembrane potential and reactive oxygen species production. Mol Med 4:165-178

38. Massari S, Frigeri S, Azzone GF 1972 Permeability to water, dimension of surface, and structural changes during swelling in rat liver mitochondria. J Membr Biol 9:67-70
39. Liang D, Hagenbuch B, Steiger B, Meier PJ 1993 Parallel decrease of $\mathrm{Na}^{+}$. taurocholate cotransport and its encoding mRNA in primary cultures of hepatocytes. Hepatology 18:1162-1166

40. Glascott PA, Glifor E, Farber JL 1992 Effects of vitamin E on the killing of cultured hepatocytes by t-butyl hydroperoxide. Mol Pharmac 41:1155-1162

41. Everson GT, Daggy B, McKinley C, Story JA 1992 Effects of psyllium hydrophilic mucilloid on LDL-cholesterol and bile acid synthesis in hypercholesterolemic man. J Lipid Res 33:1183-1192

42. Salvioli S, Ardizzoni A, Franceschi C, Cassarizza A 1997 JC-1, but not $\mathrm{DiOC}_{6}(3)$ or rhodamine 123 , is a reliable fluorescent probe to assess $\Delta \psi$ changes in intact cells: implications for studies on mitochondrial functionality during apoptosis. FEBS Lett 411:77-82

43. Bernardi P, Petronelli V 1996 The permeability transition pore as a mitochondrial calcium release channel: a critical appraisal. J Bioenerg Biomemb. 268:1005-1010

44. Neugebauer JM 1990 Detergents: an overview. In: Deutscher MP (ed) Guide to Protein Purification. Academic Press, San Diego, pp 239-82

45. Cabral DJ, Small DM 1989 Physical chemistry of bile. In: Schultz SG, Forte JG, Rauner BB (eds) Handbook of Physiology-The Gastrointestinal System III. Waverly Press, Baltimore, pp 621-662

46. Aguilar HI, Botla R, Arora AS, Bronk SF, Gores GJ 1996 Induction of the mitochondrial permeability transition by protease activity in rats: a mechanism of hepatocyte necrosis. Gastroenterology 110:558-566

47. Winklhofer-Roob BM, McKim Jr JM, Devereaux MW, Sokol RJ 1996 Characterization of site of reactive oxygen species generation in mitochondria exposed to glycochenodeoxycholic acid. Hepatology 24:338A

48. Costantini P, Chernyak BV, Petronilli V, Bernardi P 1996 Modulation of the mitochondrial permeability transition pore by pyridine nucleotides and dithiol oxidation at two separate sites. J Biol Chem 271:6746-6751

49. Fagian MM, Pereira-da-Silva L, Martins LS, Vercesi AE 1990 Membrane protein thiol cross-linking associated with the permeabilization of the inner mitochondrial membrane by $\mathrm{Ca}^{2+}$ plus prooxidants. J Biol Chem 265:19955-19960

50. Takeyama N, Matsuo N, Tanaka T 1993 Oxidative damage to mitochondria is mediated by the $\mathrm{Ca}^{2+}$-dependent inner membrane permeability transition. Biochem J 294:719-725

51. Kowaltowski AJ, Castilho RF, Vercesi AE 1996 Opening of the mitochondrial permeability transition pore by uncoupling or inorganic phosphate in the presence of $\mathrm{Ca}^{2+}$ is dependent on mitochondrial-generated reactive oxygen species. FEBS Lett 378:150-152

52. Castilho RF, Kowaltowski AJ, Meinicke AR, Bechara EJH, Vercesi AE 1995 Permeabilization of the inner mitochondrial membrane by $\mathrm{Ca}^{2+}$ ions is stimulated by $t$-butyl hydroperoxide and mediated by reactive oxygen species generated by mitochondria. Free Rad Biol Med 18:479-486

53. Bernardes CF, Meyer-Fernandes JR, Basseres DS, Castilho RF, Vercesi AE 1994 $\mathrm{Ca}^{2+}$-dependent permeabilization of the inner mitochondrial membrane by $4,4^{\prime}$ diisothiocyanato-stilbene-2,2'disulfonic acid (DIDS). Biochim Biophys Acta 1188:93-100

54. Denton RM, McCormack JG 1980 On the role of the calcium transport cycle in heart and other mammalian mitochondria. FEBS Lett 199:1-8

55. Kowaltowski AJ, Castilho RF, Vercesi AE $1995 \mathrm{Ca}^{2+}$-induced mitochondrial membrane permeabilization: role of coenzyme Q redox state. Am J Physiol 269:C141C147

56. Cai J, Jones DP 1998 Superoxide in apoptosis. Mitochondrial generation triggered by cytochrome c loss. J Biol Chem 1273:11401-11404

57. Hokanson JF, Mercier JG, Brooks GA 1995 Cyclosporine A decreases rat skeletal muscle mitochondrial respiration in vitro. Am J Respir Crit Care Med 151:1848-51

58. Imberti R, Nieminen A-L, Herman B, Lemasters JJ 1993 Mitochondrial and glycolytic dysfunction in lethal injury to hepatocytes by t-butylhydroperoxide: protection by fructose, cyclosporin and trifluoperazine. J Pharm Exp Ther 265:392-400

59. Feldman G, Haouzi D, Moreau A, Durand-Schneider A-M, Bringuier A, Berson A, Mansouri A, Fau D, Pessayre D 2000 Opening of the mitochondrial permeability transition pore causes matrix expansion and outer membrane rupture in Fas-mediated hepatic apoptosis in mice. Hepatology 31:674-683

60. Sodeman T, Bronk SF, Roberts PJ, Miyoshi H, Gores GJ 2000 Bile salts mediate hepatocyte apoptosis by increasing cell surface trafficking of Fas. Am J Physiol Gastrointest Liver Physiol 278:G992-999

61. Sokol RJ, Dahl R, Gumpricht E, Devereaux M 1998 Generation of reactive oxygen species in hepatocytes undergoing bile acid-induced apoptosis. Hepatology 28:325A(abstr)

62. Sokol RJ, Dahl R, Yerushalmi B, Gumpricht E, Devereaux MW 1999 Bile acidinduced hepatocyte apoptosis is inhibited by antioxidants and blockers of the mitochondrial permeability transition. Hepatology 30(Pt. 2):398A(abstr)

63. Benz C, Angermuller S, Otto G, Sauer P, Stremmel W, Stiehl A 2000 Effect of tauroursodeoxycholic acid on bile acid-induced apoptosis in primary human hepatocytes. Eur J Clin Invest 30:203-209

64. Anwer MS, Engelking LR, Nolan K, Sullivan D, Zimniak P, Lester R 1988 Hepatotoxic bile acids increase cytosolic calcium activity of isolated rat hepatocytes. Hepatology 8:887-891

65. Shivaram KN, Winklhofer-Roob BW, Straka MS, Devereaux MW, Everson G, Mierau GW, Sokol RJ 1998 The effect of idebenone, a coenzyme Q analogue, on hydrophobic bile acid toxicity to isolated rat hepatocytes and hepatic mitochondria. Free Rad Biol Med 25:480-492 